\title{
On functions of bounded variation on convex domains in Hilbert spaces
}

\author{
Luciana Angiuli, Simone Ferrari And Diego Pallaraid
}

\section{Dedicated to Matthias Hieber on the occasion of his 60th-Birthday}

Abstract. We study functions of bounded variation (and sets of finite perimeter) on a convex open set $\Omega \subseteq X, X$ being an infinite-dimensional separable real Hilbert space. We relate the total variation of such functions, defined through an integration by parts formula, to the short-time behaviour of the semigroup associated with a perturbation of the Ornstein-Uhlenbeck operator.

\section{Introduction}

In this paper we study some properties of functions of bounded variation ( $B V$ functions, for short) defined on an open convex subset of a real separable Hilbert space, endowed with a weighted Gaussian measure.

In finite dimension the theory of $B V$ functions is widely developed (see e.g. [3] and the references therein), whereas in the infinite-dimensional setting the analysis is still at the initial stage and many basic properties are unexplored. Besides the interest on its own, the study of $B V$ functions in infinite-dimensional spaces is motivated by problems arising in calculus of variations, stochastic analysis and it is connected with the applications in information technology (see, for example, [19,22-24,26]).

$B V$ functions for Gaussian measures in separable Banach spaces were introduced in [17] using Dirichlet forms. Inspired by the results in finite dimension, which connect the theory of functions of bounded variation to that of semigroups of bounded operators, the authors of [18] have proved an elegant characterisation of $B V$ functions in terms of the short-time behaviour of the Ornstein-Uhlenbeck semigroup. More precisely, in a separable Banach space $X$, if $\gamma$ is a centred and nondegenerate

Keywords: Infinite-dimensional analysis, Semigroups of bounded operators, Functions of bounded variation.

The authors are members of G.N.A.M.P.A. of the Italian Istituto Nazionale di Alta Matematica (INdAM). Work partially supported by the INdAM-GNAMPA Project 2019 "Metodi analitici per lo studio di PDE e problemi collegati in dimensione infinita" and by the Research project PRIN 2015 MIUR 2015233N5A "Deterministic and stochastic evolution equations". 
Gaussian measure on $X$ and $u$ belongs to the Orlicz space $L(\log L)^{1 / 2}(X, \gamma)$, then $u \in B V(X, \gamma)$ if, and only if,

$$
\liminf _{t \rightarrow 0^{+}} \int_{X}\left|D_{H} S(t) u\right|_{H} \mathrm{~d} \gamma<+\infty,
$$

where $D_{H}$ is the gradient operator along the Cameron-Martin space $H$ (see Sect. 2) and $S(t)$ is the classical Ornstein-Uhlenbeck semigroup defined via the Mehler formula (see (1)). The latter is the analogous, in the Gaussian setting, of the heat semigroup used by De Giorgi in [11] to provide the original definition of $B V$ functions in the Euclidean case. An analytic approach based on geometric measure theory is proposed in [4] to prove, as in the finite-dimensional case, the equivalence of different definitions of $B V(X, \gamma)$ functions also, as in [18], in terms of the Ornstein-Uhlenbeck semigroup $S(t)$ near $t=0$. Similar De Giorgi-type characterisations of $B V$ functions have been obtained for weighted Gaussian measures and more recently for general Fomin differentiable measures in Hilbert spaces, see [12] and the reference therein.

Beside the difficulty of considering general measures, another difficulty of different nature comes from the consideration of functions defined in domains rather than in the whole space. These difficulties come from the lack of factorisation of the underlying measure (that is lost even for Gaussian measures in domains) and the unavailability of decomposition of the domain through the classical method of local charts. Therefore, the easiest interesting case seems to be that of convex domains that are possible to deal with through global penalisation techniques. This is the approach we followed in [6] (see also [21]), and in this paper we take advantage of the results proved there. We start from a weighted Gaussian measure $v:=e^{-U} \gamma$ in a Hilbert space $X$, where $U: X \rightarrow \mathbb{R}$ is convex and sufficiently regular, and consider an open convex domain $\Omega \subseteq X$. After introducing the Cameron-Martin space $H$ and the Malliavin gradient $D_{H}$ along it, we define the form $(u, v) \mapsto \int_{\Omega}\left\langle D_{H} u, D_{H} v\right\rangle_{H} \mathrm{~d} v$ on the appropriate Sobolev spaces. The perturbed Ornstein-Uhlenbeck operator $L_{\Omega}$ is then defined in the usual variational way, and it is the generator of an analytic, strongly continuous and contraction semigroup $T_{\Omega}(t)$ in $L^{p}(\Omega, v)$, for $1<p<\infty$.

For the latter, differently from the Ornstein-Uhlenbeck semigroup in the whole space, no explicit integral representation which allows for direct computations is known. In this direction, in [21] the authors consider the restrictions to an open convex set $\Omega \subseteq X$ of $B V(X, \gamma)$ functions and they characterise the finiteness of their total variation in $\Omega$ in terms of the Neumann Ornstein-Uhlenbeck semigroup defined in $\Omega$.

Following the ideas in [2], we define the $B V(\Omega, v)$ space through an integration by parts formula against suitable Lipschitz functions. Then, we show that the functions $u$ of bounded variation in $\Omega$ with respect to $v$ can be characterised by the finiteness of the limit of $\left\|D_{H} T_{\Omega}(t) u\right\|_{L^{1}(\Omega, v ; H)}$ as $t \rightarrow 0^{+}$. The proof of this result relies on a commutation formula between the semigroup $T_{\Omega}(t)$ and the gradient operator along $H$ (see Proposition 5). This result was already known in the case of the whole space (see [12]). Here, by means of the crucial pointwise gradient estimate (7) and suitable 
penalisations $\Phi_{\varepsilon}$ of $U$ outside $\Omega$ based on the distance function from $\Omega$ along $H$ (here is a first point where the convexity of $\Omega$ comes into the play) and the penalisation $v_{\varepsilon}:=e^{-\Phi_{\varepsilon}} \gamma$ of the measure $v$, see Sect. 2.1, we are able to let $\varepsilon$ to $0^{+}$and to come back to $\Omega$.

Finally, we provide a necessary condition in order that a set $E$ is of finite perimeter in $\Omega$ with respect to $v$ (i.e. $\chi_{E} \in B V(\Omega, v)$ ). This condition is given in terms of the short-time behaviour of the Ornstein-Uhlenbeck content $\left\|T_{\Omega}(t) \chi_{E}-\chi_{E}\right\|_{L^{1}(\Omega, v)}$ as $t \rightarrow 0^{+}$. Further, a sufficient condition in terms of a related quantity is also shown. This circle of ideas goes back to [20], which originated several researches. Among these, the only infinite-dimensional result, proved for $B V$ functions in space endowed with a Gaussian measure, is in [5].

\section{Hypotheses and preliminaries}

Let $H_{1}$ and $H_{2}$ be two real Hilbert spaces with inner products $\langle\cdot, \cdot\rangle_{H_{1}}$ and $\langle\cdot, \cdot\rangle_{H_{2}}$, respectively. We denote by $\mathcal{B}\left(H_{1}\right)$ the $\sigma$-algebra of Borel subsets of $H_{1}$ and by $C_{b}^{k}\left(H_{1} ; H_{2}\right), k \in \mathbb{N} \cup\{\infty\}$ the set of $k$-times Fréchet differentiable functions from $H_{1}$ to $H_{2}$ with bounded derivatives up to order $k\left(C_{b}^{k}\left(H_{1}\right)\right.$ if $\left.H_{2}=\mathbb{R}\right)$. For $\Phi \in C_{b}^{1}\left(H_{1} ; H_{2}\right)$ we denote by $D \Phi(x)$ the derivative of $\Phi$ at $x \in H_{1}$ : if $f \in C_{b}^{1}\left(H_{1}\right)$, for every $x \in H_{1}$ there exists a unique $k \in H_{1}$ such that $D f(x)(h)=\langle h, k\rangle_{H_{1}}, h \in H_{1}$ and we set $D f(x):=k$. Let $X$ be a separable Hilbert space, with inner product $\langle\cdot, \cdot\rangle$ and norm $|\cdot|$. Let $B \in \mathcal{L}(X)$ (the set of bounded linear operators from $X$ to itself). We say that $B$ is non-negative if $\langle B x, x\rangle \geq 0$ for every $x \in X$ and positive if $\langle B x, x\rangle>0$ for every $x \in X \backslash\{0\}$. We recall that a non-negative and self-adjoint operator $B \in \mathcal{L}(X)$ is a trace class operator whenever $\operatorname{Tr}(B):=\sum_{n=1}^{\infty}\left\langle B e_{n}, e_{n}\right\rangle<\infty$ for some (and hence, every) orthonormal basis $\left(e_{n}\right)_{n \in \mathbb{N}}$ of $X$.

Let $\gamma$ be a nondegenerate Gaussian measure on $X$ with mean zero and covariance operator $Q_{\infty}:=-Q A^{-1}$, where the operators $Q$ and $A$ satisfy the following assumptions.

Hypotheses 1. (i) $Q \in \mathcal{L}(X)$ is a self-adjoint and non-negative operator with Ker $Q=\{0\}$

(ii) $A: D(A) \subseteq X \rightarrow X$ is a self-adjoint operator satisfying $\langle A x, x\rangle \leq-\omega|x|^{2}$ for every $x \in D(A)$ and some positive $\omega$;

(iii) $Q e^{t A}=e^{t A} Q$ for any $t \geq 0$;

(iv) $\operatorname{Tr}\left(-Q A^{-1}\right)<\infty$.

Under Hypotheses 1(i)-(iii), the measure $\gamma$ is well defined and the OrnsteinUhlenbeck semigroup defined via the Mehler formula

$$
(S(t) f)(x):=\int_{X} f\left(e^{-t} x+\sqrt{1-e^{-2 t}} y\right) \mathrm{d} \gamma(y), \quad x \in X, f \in L^{1}(X, \gamma)
$$

is symmetric in $L^{2}(X, \gamma)$. We fix an orthonormal basis $\left(v_{k}\right)_{k \in \mathbb{N}}$ of $X$ such that

$$
Q_{\infty} v_{k}=\lambda_{k} v_{k}, \quad k \in \mathbb{N},
$$


where $\left(\lambda_{k}\right)_{k \in \mathbb{N}}$ is the decreasing sequence of eigenvalues of $Q_{\infty}$. Under Hypothesis 1(iv), the Cameron-Martin space $\left(H,|\cdot|_{H}\right)$

$$
H:=Q_{\infty}^{1 / 2}(X)=\left\{x \in X \mid \sum_{k=1}^{\infty} \lambda_{k}^{-1}\left\langle x, v_{k}\right\rangle^{2}<\infty\right\},
$$

where $|\cdot|_{H}$ is induced by the inner product $\langle h, k\rangle_{H}:=\left\langle Q_{\infty}^{-1 / 2} h, Q_{\infty}^{-1 / 2} k\right\rangle$, is a Hilbert space compactly and densely embedded in $X$ (see [8] and [13] for further details). The sequence $\left(e_{k}\right)_{k \in \mathbb{N}}$, where $e_{k}=\sqrt{\lambda_{k}} v_{k}$ for any $k \in \mathbb{N}$, is an orthonormal basis of $H$. By Hypotheses 1 , the operator $-Q_{\infty}^{-1}: D\left(Q_{\infty}^{-1}\right) \subseteq X \rightarrow X\left(-Q_{\infty}^{-1}: D\left(Q_{\infty}^{-1}\right) \subseteq H \rightarrow\right.$ $H$, respectively) is the generator of a contractive and strongly continuous semigroup $e^{-t Q_{\infty}^{-1}}$ on $X$ (on $H$, respectively), see [14, Proposition p. 84]). If $Y$ is a Banach space with norm $\|\cdot\|_{Y}$, a function $F: X \rightarrow Y$ is said to be $H$-Lipschitz continuous if there exists a positive constant $C$ such that

$$
\|F(x+h)-F(x)\|_{Y} \leq C|h|_{H},
$$

for every $h \in H$ and $\gamma$-a.e. $x \in X$. We denote by [F] $H$-Lip the best constant $C$ in (3). For more information see [8, Sections 4.5 and 5.11]. We denote by $\mathcal{H}_{2}$ the space of the Hilbert-Schmidt operators in $H$ that is the space of the bounded linear operators $B: H \rightarrow H$ such that $\|B\|_{\mathcal{H}_{2}}^{2}:=\sum_{i=1}^{\infty}\left|B g_{i}\right|_{H}^{2}$ is finite, where $\left\{g_{n} \mid n \in \mathbb{N}\right\}$ is any orthonormal basis of $H$. We say that $f: X \rightarrow \mathbb{R}$ is $H$-differentiable at $x_{0} \in X$ if there exists $\ell \in H$ such that

$$
f\left(x_{0}+h\right)=f\left(x_{0}\right)+\langle\ell, h\rangle_{H}+o\left(|h|_{H}\right), \quad \text { as }|h|_{H} \rightarrow 0 .
$$

In such a case we set $D_{H} f\left(x_{0}\right):=\ell$ and $D_{i} f\left(x_{0}\right):=\left\langle D_{H} f\left(x_{0}\right), e_{i}\right\rangle_{H}$ for any $i \in \mathbb{N}$. The derivative $D_{H} f\left(x_{0}\right)$ is called the Malliavin derivative of $f$ at $x_{0}$. In a similar way we say that $f$ is twice $H$-differentiable at $x_{0}$ if $f$ is $H$-differentiable near $x_{0}$ and there exists $\mathcal{B} \in \mathcal{H}_{2}$ such that

$f\left(x_{0}+h\right)=f\left(x_{0}\right)+\left\langle D_{H} f\left(x_{0}\right), h\right\rangle_{H}+\frac{1}{2}\langle\mathcal{B} h, h\rangle_{H}+o\left(|h|_{H}^{2}\right), \quad$ as $|h|_{H} \rightarrow 0$.

In such a case we set $D_{H}^{2} f\left(x_{0}\right):=\mathcal{B}$ and $D_{i j} f\left(x_{0}\right):=\left\langle D_{H}^{2} f\left(x_{0}\right) e_{j}, e_{i}\right\rangle_{H}$ for any $i, j \in \mathbb{N}$. If $f$ is twice $H$-differentiable at $x_{0}$, then $D_{i j} f\left(x_{0}\right)=D_{j i} f\left(x_{0}\right)$ for every $i, j \in \mathbb{N}$. Notice that if $f: X \rightarrow \mathbb{R}$ is once or twice Fréchet differentiable at $x_{0}$, then it is once or twice $H$-differentiable at $x_{0}$ and it holds $D_{H} f\left(x_{0}\right)=Q_{\infty} D f\left(x_{0}\right)$, and $D_{H}^{2} f\left(x_{0}\right)=Q_{\infty} D^{2} f\left(x_{0}\right) Q_{\infty}$, where the equality must be understood as holding in $H$. For any $k \in \mathbb{N} \cup\{\infty\}$, we denote by $\mathcal{F} C_{b}^{k}(X)$, the space of cylindrical $C_{b}^{k}$ functions, i.e. the set of functions $f: X \rightarrow \mathbb{R}$ such that $f(x)=\varphi\left(\left\langle x, h_{1}\right\rangle, \ldots,\left\langle x, h_{N}\right\rangle\right)$ for some $\varphi \in C_{b}^{k}\left(\mathbb{R}^{N}\right), h_{1}, \ldots, h_{N} \in H$ and $N \in \mathbb{N}$. By $\mathcal{F} C_{b}^{k}(X, H)$ we denote $H$-valued cylindrical $C_{b}^{k}$ functions with finite rank. The Sobolev spaces in the sense of Malliavin $D^{1, p}(X, \gamma)$ and $D^{2, p}(X, \gamma)$ with $p \in[1, \infty)$ are defined as the completions of the smooth cylindrical functions $\mathcal{F} C_{b}^{\infty}(X)$ in the norms

$$
\|f\|_{D^{1, p}(X, \gamma)}:=\left(\|f\|_{L^{p}(X, \gamma)}^{p}+\int_{X}\left|D_{H} f\right|_{H}^{p} \mathrm{~d} \gamma\right)^{\frac{1}{p}} ;
$$




$$
\|f\|_{D^{2, p}(X, \gamma)}:=\left(\|f\|_{D^{1, p(X, \gamma)}}^{p}+\int_{X}\left\|D_{H}^{2} f\right\|_{\mathcal{H}_{2}}^{p} \mathrm{~d} \gamma\right)^{\frac{1}{p}}
$$

This is equivalent to considering the domain of the closure of the gradient operator, defined on smooth cylindrical functions, in $L^{p}(X, \gamma)$ (see [8, Section 5.2]). Let $U$ : $X \rightarrow \mathbb{R}$ satisfy the following assumptions.

Hypotheses 2. $U$ is a convex function which belongs to $C^{2}(X) \cap D^{1, q}(X, \gamma)$ for all $q \in[1, \infty)$ with $H$-Lipschitz gradient.

The convexity of the function $U$ guarantees that $U$ is bounded from below by a linear function, and therefore, it decreases at most linearly and by Fernique theorem (see [8, Theorem 2.8.5]) $e^{-U}$ belongs to $L^{1}(X, \gamma)$. Then, we can consider the finite log-concave measure

$$
v:=e^{-U} \gamma
$$

It is obvious that $\gamma$ and $v$ are equivalent measures, hence saying that a statement holds $\gamma$-a.e. is the same as saying that it holds $\nu$-a.e. Moreover as $U \in \cap_{q \geq 1} D^{1, q}(X, \gamma)$, the operator $D_{H}: \mathcal{F} C_{b}^{1}(X) \rightarrow L^{p}(X, v ; H)$ is closable in $L^{p}(X, v), p \in(1, \infty)$ and the space $D^{1, p}(X, v), p>1$ can be defined as the domain of its closure (still denoted by $D_{H}$ ). In a similar way we may define $D^{2, p}(X, v), p \in(1, \infty)$ (for more details see $[1,9,16])$. The Gaussian integration by parts formula $\int_{X} D_{i} f \mathrm{~d} \gamma=\frac{1}{\sqrt{\lambda_{i}}} \int_{X}\left\langle x, v_{i}\right\rangle f \mathrm{~d} \gamma$, which holds true for any $f \in \mathcal{F} C_{b}^{1}(X)$ and $i \in \mathbb{N}$, yields

$$
\int_{X} \psi D_{i} \varphi \mathrm{d} \nu+\int_{X} \varphi D_{i} \psi \mathrm{d} v=\int_{X} \varphi \psi D_{i} U \mathrm{~d} \nu+\frac{1}{\sqrt{\lambda_{i}}} \int_{X}\left\langle x, v_{i}\right\rangle \varphi \psi \mathrm{d} v, \quad i \in \mathbb{N},
$$

for any $\varphi \in D^{1, p}(X, v)(p>1)$ and $\psi \in \mathcal{F} C_{b}^{1}(X)$.

In what follows $\Omega$ denotes an open subset of $X$. In this case, the spaces $D^{1, p}(\Omega, v)$ and $D^{2, p}(\Omega, v), p \in(1, \infty)$, can be defined in a similar way as in the whole space, thanks to the following result (see [6, Proposition 1.4]).

Proposition 1. Assume that Hypotheses 1 and 2 are satisfied. Let $p \in(1, \infty)$ and let $\Omega$ be an open subset of $X$. The operators $D_{H}: \mathcal{F} C_{b}^{\infty}(\Omega) \rightarrow L^{p}(\Omega, v ; H)$ and

$$
\left(D_{H}, D_{H}^{2}\right): \mathcal{F} C_{b}^{\infty}(\Omega) \times \mathcal{F} C_{b}^{\infty}(\Omega) \rightarrow L^{p}(\Omega, v ; H) \times L^{p}\left(\Omega, v ; \mathcal{H}_{2}\right)
$$

are closable in $L^{p}(\Omega, v)$ and $L^{p}(\Omega, v) \times L^{p}(\Omega, v)$, respectively. Here $\mathcal{F} C_{b}^{\infty}(\Omega)$ is the space of the restrictions to $\Omega$ of functions in $\mathcal{F} C_{b}^{\infty}(X)$.

The spaces $D^{1, p}(\Omega, v ; H), p \in(1, \infty)$, are defined in a similar way, replacing smooth cylindrical functions with $H$-valued smooth cylindrical functions with finite rank. We recall that if $F \in D^{1, p}(\Omega, v ; H)$, then $D_{H} F(x)$ belongs to $\mathcal{H}_{2}$ for a.e. $x \in \Omega$. We denote by $p^{\prime}$ the conjugate exponent to $p \in(1, \infty)$. 
2.1. Perturbed Ornstein-Uhlenbeck semigroup on convex domains

In order to consider the initial boundary value problems defined in $\Omega$ we define the distance function along $H$

$$
d_{\Omega}(x):= \begin{cases}\inf \left\{|h|_{H} \mid h \in H \cap(\Omega-x)\right\}, & H \cap(\Omega-x) \neq \emptyset \\ \infty, & H \cap(\Omega-x)=\emptyset,\end{cases}
$$

for $x \in X$, and we recall some useful regularity results, (see, for instance, [8, Theorems 2.8.5 and 5.11.2] and [10, Section 3]).

Proposition 2. If $\Omega \subseteq X$ is an open convex set, then $d_{\Omega}^{2}$ is $H$-differentiable and its Malliavin derivative is $H$-Lipschitz with $H$-Lipschitz constant less than or equal to 2 , i.e.

$$
\left|D_{H} d_{\Omega}^{2}(x+h)-D_{H} d_{\Omega}^{2}(x)\right|_{H} \leq 2|h|_{H},
$$

for any $h \in H$ and for v-a.e $x \in X$. Moreover, $D_{H}^{2} d_{\Omega}^{2}$ exists v-a.e. in $X$ and $d_{\Omega}^{2}$ belongs to $D^{2, p}(X, v)$ for every $p \in[1, \infty)$.

We require some further regularity on $d_{\Omega}^{2}$.

Hypotheses 3. Let $\Omega$ be an open convex subset of $X$ such that $\nu(\partial \Omega)=0$ and $D_{H}^{2} d_{\Omega}^{2}$ is $H$-continuous $\gamma$-a.e. in $X$, i.e. for $\gamma$-a.e. $x \in X$ we have

$$
\lim _{|h|_{H} \rightarrow 0} D_{H}^{2} d_{\Omega}^{2}(x+h)=D_{H}^{2} d_{\Omega}^{2}(x) .
$$

Remark 1. As stated in [6, Remark 1.7] there is a rather large class of subsets of $X$ satisfying Hypothesis 3. For instance, if $\partial \Omega$ is (locally) a $C^{2}$-embedding in $X$ of an open subset of a hyperplane in $X$ and $v(\partial \Omega)=0$, then Hypothesis 3 is satisfied. Easy examples are open balls and open ellipsoids of $X$, open hyperplanes of $X$ and every set of the form $\Omega=\{x \in X \mid G(x)<0\}$, where $G: X \rightarrow \mathbb{R}$ is a $C^{2}$-convex function such that $D_{H} G$ is nonzero at every point of $\partial \Omega$.

We consider the semigroup $T_{\Omega}(t)$ on $L^{2}(\Omega, v)$ and its generator $L_{\Omega}$ :

$$
\begin{gathered}
D\left(L_{\Omega}\right)=\left\{u \in D^{1,2}(\Omega, v) \mid \exists v \in L^{2}(\Omega, v)\right. \text { such that } \\
\left.\int_{\Omega}\left\langle D_{H} u, D_{H} \varphi\right\rangle_{H} \mathrm{~d} v=-\int_{\Omega} v \varphi \mathrm{d} v \forall \varphi \in \mathcal{F} C_{b}^{\infty}(\Omega)\right\}
\end{gathered}
$$

with $L_{\Omega} u:=v$ if $u \in D\left(L_{\Omega}\right)$. We recall (see [6, Section 2]) an approximation procedure of $T_{\Omega}(t) f$, when $f \in L^{2}(\Omega, v)$, through $\mathcal{F} C_{b}^{3}(X)$ functions that relies on a reduction to a finite (say $n$-) dimensional space and on a $\varepsilon$-penalisation argument. Accordingly, the approximation depends on two parameters $n$ and $\varepsilon$. More precisely, we consider the function $\Phi_{\varepsilon}: X \rightarrow \mathbb{R}$ defined by

$$
\Phi_{\varepsilon}(x):=U(x)+\frac{1}{2 \varepsilon} d_{\Omega}^{2}(x), \quad x \in X, \varepsilon>0,
$$


and the measure $v_{\varepsilon}$ given by $e^{-\Phi_{\varepsilon}} \gamma$. Next, we consider the operator $L_{\varepsilon}$ on the whole $X$ defined as

$$
\begin{aligned}
& D\left(L_{\varepsilon}\right)=\left\{u \in D^{1,2}\left(X, v_{\varepsilon}\right) \mid \exists v \in L^{2}\left(X, v_{\varepsilon}\right)\right. \text { such that } \\
& \left.\quad \int_{X}\left\langle D_{H} u, D_{H} \varphi\right\rangle_{H} \mathrm{~d} v_{\varepsilon}=-\int_{X} v \varphi \mathrm{d} v_{\varepsilon} \text { for every } \varphi \in \mathcal{F} C_{b}^{\infty}(X)\right\},
\end{aligned}
$$

with $L_{\varepsilon} u:=v$ if $u \in D\left(L_{\varepsilon}\right)$, and the semigroup $T_{\varepsilon}(t)$ generated by $L_{\varepsilon}$ in $L^{2}\left(X, v_{\varepsilon}\right)$. We point out that $L_{\varepsilon}$ acts on smooth cylindrical functions $\varphi$ as follows

$$
\begin{aligned}
L_{\varepsilon} \varphi & =\operatorname{Tr}\left(D_{H}^{2} \varphi\right)-\sum_{i=1}^{\infty} \lambda_{i}^{-1}\left\langle x, e_{i}\right\rangle D_{i} \varphi-\left\langle D_{H} \Phi_{\varepsilon}, D_{H} \varphi\right\rangle_{H} \\
& =\operatorname{Tr}\left(D_{H}^{2} \varphi\right)-\sum_{i=1}^{\infty} \lambda_{i}^{-1}\left\langle x, e_{i}\right\rangle D_{i} \varphi-\left\langle D_{H} U+\frac{1}{2 \varepsilon} D_{H} d_{\Omega}^{2}, D_{H} \varphi\right\rangle_{H}
\end{aligned}
$$

Now we recall a useful approximation result whose proof can be found in [6, Theorem $2.8]$.

Theorem 1. Under Hypotheses 1, 2 and 3 the following statements hold true.

(i) For any $\varepsilon>0$ and $f \in L^{2}\left(X, v_{\varepsilon}\right)$, there exists a sequence $\left(f_{n}\right)_{n \in \mathbb{N}} \subseteq L^{2}\left(X, v_{\varepsilon}\right)$ converging to $f$ in $L^{2}\left(X, v_{\varepsilon}\right)$ such that $T_{\varepsilon}(t) f_{n}$ is in $\mathcal{F} C_{b}^{3}(X)$ and

$$
\lim _{n \rightarrow \infty}\left\|T_{\varepsilon}(t) f_{n}-T_{\varepsilon}(t) f\right\|_{D^{1,2}\left(X, v_{\varepsilon}\right)}=0, \quad t>0 .
$$

In addition, if $f \in D^{1,2}\left(X, v_{\varepsilon}\right)$ then the sequence $\left(f_{n}\right)$ can be chosen in a way that $D_{H} f_{n}$ converges to $D_{H} f$ in $L^{1}\left(X, v_{\varepsilon} ; H\right)$, as $n \rightarrow \infty$.

(i) For any $f \in L^{2}(\Omega, v)$ there exists an infinitesimal sequence $\left(\varepsilon_{n}\right)_{n \in \mathbb{N}}$ such that $T_{\varepsilon_{n}}(t) \widetilde{f}$ weakly converges to $T_{\Omega}(t) f$ in $D^{1,2}(\Omega, v)$, where $\tilde{f}$ is any $L^{2}$-extension of $f$ to $X$.

We collect some properties of $T_{\Omega}(t)$, see [6, Proposition 1.10, Theorems $3.1 \& 3.3$ ].

Proposition 3. If Hypotheses 1, 2 and 3 hold true, then

(i) the semigroup $T_{\Omega}(t)$ generated in $L^{2}(\Omega, v)$ can be extended to a positivity preserving contraction semigroup in $L^{p}(\Omega, v)$ for every $1 \leq p \leq \infty$ and $t \geq 0$, still denoted by $T_{\Omega}(t)$. It is strongly continuous in $L^{p}(\Omega, v)$ for any $p \in[1, \infty)$ and consistent;

(ii) for any $p \in[1, \infty), f \in L^{p}(\Omega, v)$ and $g \in L^{\infty}(\Omega, v)$ it holds

$$
\int_{\Omega} f T_{\Omega}(t) g \mathrm{~d} \nu=\int_{\Omega} g T_{\Omega}(t) f \mathrm{~d} \nu, \quad t>0 ;
$$

(iii) for any $p \in(1, \infty), f \in L^{p}(\Omega, v)$ and $t>0$ there is $K_{p}>0$ such that

$$
\left|D_{H} T_{\Omega}(t) f\right|_{H}^{p} \leq K_{p} t^{-p / 2} T_{\Omega}(t)|f|^{p} \quad \text { v-a.e. in } \Omega ;
$$


(iv) if $f \in D^{1, p}(\Omega, v), t>0$ and $p \in[1, \infty)$ it holds

$$
\left|D_{H} T_{\Omega}(t) f\right|^{p} \leq e^{-p \lambda_{1}^{-1} t} T_{\Omega}(t)\left|D_{H} f\right|_{H}^{p} \quad \text { v-a.e. in } \Omega \text {. }
$$

We point out that the results in Proposition 3 continue to hold if we replace $\Omega, v$ and $T_{\Omega}(t)$ by $X, v_{\varepsilon}$ and $T_{\varepsilon}(t)$, respectively.

2.2. BV functions in Hilbert spaces: definitions and some known facts

We introduce $B V$ functions in the Wiener space setting. Let $Y$ be a separable Hilbert space with norm $|\cdot|_{Y}$. We recall that in a separable space $X$ the $\sigma$-algebra $\mathcal{B}(X)$ is generated by the family of the cylindrical sets (see e.g. [25]). Denote by $\mathcal{M}(\Omega ; Y$ ) the set of Borel $Y$-valued measures on $\Omega$. If $Y=\mathbb{R}$ then we write $\mathcal{M}(\Omega)$. The total variation of $\mu \in \mathcal{M}(\Omega ; Y)$ is the positive Borel measure

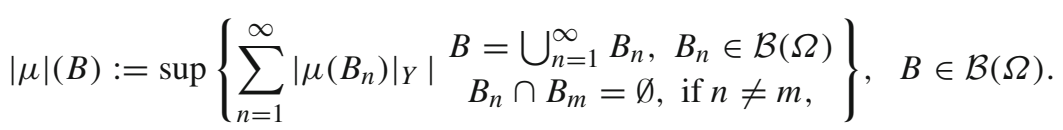

Let $\operatorname{Lip}_{c}(\Omega ; Y)$ be the set of bounded Lipschitz continuous $Y$-valued functions $g$ : $\Omega \rightarrow Y$ such that dist(supp $g, X \backslash \Omega)>0$ and define the space $B V(\Omega, v)$ as follows.

Definition 1. Let $\Omega$ be an open subset of $X$. We say that a function $f \in L^{2}(\Omega, v)$ is of bounded variation in $\Omega$, and we write $f \in B V(\Omega, v)$, if there exists a measure $\mu \in \mathcal{M}(\Omega ; H)$ such that

$$
\int_{\Omega} f \partial_{h}^{*} g \mathrm{~d} \nu=-\int_{\Omega} g d\langle\mu, h\rangle_{H},
$$

for every $g \in \operatorname{Lip}_{c}(\Omega)$ and $h \in H$, where $\partial_{h}^{*}$ denotes, up to the sign, the adjoint in $L^{2}(\Omega, v)$ of the partial derivative along $h \in H$. In this case we set $D_{v} f:=\mu$.

As in the finite-dimensional case, one can characterise functions of bounded variation by their total variation.

Definition 2. Let $\Omega$ be an open subset of $X$ and $u \in L^{2}(\Omega, v)$. We define the variation of $u$ in $\Omega$ by

$$
V_{v}(u, \Omega):=\sup \left\{\begin{array}{l}
\int_{\Omega} u \operatorname{div}_{v} g \mathrm{~d} v \mid \begin{array}{c}
F \subseteq H \text { finite-dimensional, } \\
g \in \operatorname{Lip}_{c}(\Omega ; F),\|g\|_{\infty} \leq 1 .
\end{array}
\end{array}\right\} .
$$

Here $\operatorname{div}_{\mathcal{v}} g=\sum_{i=1}^{N} \partial_{k_{i}}^{*} g_{i}(x)$ if $g(x)=\sum_{i=1}^{N} g_{i}(x) k_{i}$ and $F=\operatorname{span}\left\{k_{1}, \ldots, k_{N}\right\}$ for some $N \in \mathbb{N}$.

When $\Omega=X$, in the two definitions above we can consider $\operatorname{Lip}_{b}(X)$ and $\operatorname{Lip}_{b}(X ; F)$, respectively, as test functions spaces.

As announced, in [2, Theorem 5.7] it has been proved that $u \in B V(\Omega, v)$ if and only if $V_{v}(u, \Omega)$ is finite. Moreover, in this case

$$
\left|D_{\nu} u\right|(\Omega)=V_{v}(u, \Omega) .
$$


Finally we say that a Borel subset $E$ of $X$ is of finite perimeter in $\Omega$ with respect to $v$, whenever the function $\chi_{E}$ belongs to $B V(\Omega, v)$. In this case we denote by $P_{v}(E, \Omega)$ the total variation of $\chi_{E}$ in $\Omega$.

\section{A De Giorgi type characterisation}

The main result of this section is the De Giorgi type characterisation of $B V(\Omega, v)$ functions in Theorem 3, which relies on a "quasi-commutative" formula between the semigroup $T_{\Omega}(t)$ and the $H$-gradient operator $D_{H}$; here estimate (8) plays a crucial role. This formula is inspired by an analogous formula proved in [12]. We first define the Sobolev spaces $D^{1,2}\left(X, v_{\varepsilon} ; H\right)$.

Definition 3. We denote by $D^{1,2}\left(X, v_{\varepsilon} ; H\right)$ the domain of the closure of the operator $D_{H}: \mathcal{F} C_{b}^{1}(X, H) \rightarrow L^{2}\left(X, v_{\varepsilon} ; \mathcal{H}_{2}\right)$ in the $L^{2}\left(X, v_{\varepsilon} ; H\right)$ norm (see [7, Section 8.1]). $D_{H}$ is defined as

$$
D_{H} \Phi(x)=\sum_{i=1}^{n} \sum_{j=1}^{k(i)} \frac{\partial \varphi_{i}}{\partial \xi_{j}}\left(\left\langle x, x_{1}\right\rangle, \ldots,\left\langle x, x_{k(i)}\right\rangle\right)\left(\left(Q_{\infty}^{1 / 2} x_{j}\right) \otimes e_{i}\right),
$$

where $\left\{e_{i} \mid i \in \mathbb{N}\right\}$ is an orthonormal basis of $H$ and

$$
\Phi(x)=\sum_{i=1}^{n} \varphi_{i}\left(\left\langle x, x_{1}\right\rangle, \ldots,\left\langle x, x_{k(i)}\right\rangle\right) e_{i}
$$

for some $n \in \mathbb{N}, k(i) \in \mathbb{N}, x_{1}, \ldots, x_{k(i)} \in X$ and $\varphi_{i} \in C_{b}^{1}\left(\mathbb{R}^{k(i)}\right)$ for every $i=$ $1, \ldots, n$. In an analogous way we define the space $D^{1,2}(\Omega, v ; H)$.

We first show a vector-valued version of Theorem 1 . Let $\mathbf{L}_{\varepsilon}$ in $L^{2}\left(X, v_{\varepsilon} ; H\right)$ be the operator defined via the quadratic form by

$$
(F, G) \mapsto \int_{X}\left\langle D_{H} F, D_{H} G\right\rangle_{\mathcal{H}_{2}} \mathrm{~d} v_{\varepsilon} \quad F, G \in D^{1,2}\left(X, v_{\varepsilon} ; H\right) .
$$

In the same way we define the operator $\mathbf{L}_{\Omega}$ in in $L^{2}(\Omega, v ; H)$. We recall that by [14, p. 84] ( [14, Corollary 3.17 and Proposition 3.23] and [14, Corollary 4.8], respectively), the operators $\mathbf{L}_{\varepsilon}$ and $\mathbf{L}_{\Omega}$ generate strongly continuous semigroups $\mathbf{T}_{\varepsilon}(t)$ and $\mathbf{T}_{\Omega}(t)$ (contractive and analytic, respectively).

Proposition 4. The operators $\mathbf{L}_{\varepsilon}, \mathbf{L}_{\Omega}$ and the semigroups $\mathbf{T}_{\varepsilon}(t)$ and $\mathbf{T}_{\Omega}(t)$ act component by component, i.e. if $F \in D\left(\mathbf{L}_{\varepsilon}\right)\left(D\left(\mathbf{L}_{\Omega}\right)\right.$, respectively), and it is such that $F=\sum_{i=1}^{\infty} f_{i} e_{i}$ for some basis $\left\{e_{n} \mid n \in \mathbb{N}\right\}$ of $H$, then $f_{i} \in D\left(L_{\varepsilon}\right)\left(D\left(L_{\Omega}\right)\right.$, respectively) and

$$
\mathbf{L}_{\varepsilon} F=\sum_{i=1}^{\infty}\left(L_{\varepsilon} f_{i}\right) e_{i}, \quad \mathbf{L}_{\Omega} F=\sum_{i=1}^{\infty}\left(L_{\Omega} f_{i}\right) e_{i} .
$$


Moreover, for every $t>0$, if $F \in L^{2}\left(X, v_{\varepsilon} ; H\right)\left(L^{2}(\Omega, v ; H)\right.$, respectively), and it is such that $F=\sum_{i=1}^{\infty} f_{i} e_{i}$ for some basis $\left\{e_{n} \mid n \in \mathbb{N}\right\}$ of $H$ and $f_{i} \in L^{2}\left(X, v_{\varepsilon}\right)$ $\left(L^{2}(\Omega, v)\right.$, respectively) then

$$
\mathbf{T}_{\varepsilon}(t) F=\sum_{i=1}^{\infty}\left(T_{\varepsilon}(t) f_{i}\right) e_{i}, \quad\left(\mathbf{T}_{\Omega}(t) F=\sum_{i=1}^{\infty}\left(T_{\Omega}(t) f_{i}\right) e_{i}, \text { respectively }\right) .
$$

The above identities hold $v_{\varepsilon}$-a.e. in $X\left(v_{\Omega}\right.$-a.e. in $\Omega$, respectively).

Proof. We only show the results for $\mathbf{L}_{\varepsilon}$ and $\mathbf{T}_{\varepsilon}(t)$. Let $F=\sum_{i=1}^{\infty} f_{i} e_{i} \in D\left(\mathbf{L}_{\varepsilon}\right)$ and let $G=g e_{j}$ for some $j \in \mathbb{N}$ and $g \in D^{1,2}\left(X, v_{\varepsilon}\right)$; then

$$
\begin{aligned}
\int_{X}\left\langle D_{H} g, D_{H} f_{j}\right\rangle_{H} \mathrm{~d} v_{\varepsilon} & =\int_{X}\left\langle D_{H} G, D_{H} F\right\rangle_{\mathcal{H}_{2}} \mathrm{~d} v_{\varepsilon} \\
& =-\int_{X}\left\langle G, \mathbf{L}_{\varepsilon} F\right\rangle_{H} \mathrm{~d} v_{\varepsilon}=-\int_{X} g\left(\mathbf{L}_{\varepsilon} F\right)_{j} \mathrm{~d} v_{\varepsilon} .
\end{aligned}
$$

This shows that $f_{j} \in D\left(L_{\varepsilon}\right)$ (see (5)) and $L_{\varepsilon} f_{j}=\left(\mathbf{L}_{\varepsilon} F\right)_{j}$. Now observe that

$$
D_{t}\left(\mathbf{T}_{\varepsilon}(t) F\right)_{j}=\left(\mathbf{L}_{\varepsilon} \mathbf{T}_{\varepsilon}(t) F\right)_{j}=L_{\varepsilon}\left(\mathbf{T}_{\varepsilon}(t) F\right)_{j}, \quad\left(\mathbf{T}_{\varepsilon}(0) F\right)_{j}=f_{j}
$$

Thus, by the uniqueness of the solution of the Cauchy problem associated with $D_{t}-L_{\varepsilon}$ in $L^{2}\left(X, v_{\varepsilon}\right)$, it follows that $\left(\mathbf{T}_{\varepsilon}(t) F\right)_{j}=T_{\varepsilon}(t) f_{j}$ for any $t>0$. The arbitrariness of $j \in \mathbb{N}$ concludes the proof.

Remark 2. According to the definition of $\mathbf{T}_{\varepsilon}(t)$ and $\mathbf{T}_{\Omega}(t)$ it is immediately seen that for every $F \in L^{2}\left(X, v_{\varepsilon} ; H\right)$ and $G \in L^{2}(\Omega, v ; H)$

$$
\left|\mathbf{T}_{\varepsilon}(t) F\right|^{2} \leq T_{\varepsilon}(t)|F|^{2}, \quad t \geq 0, v_{\varepsilon} \text {-a.e. in } X
$$

and

$$
\left|\mathbf{T}_{\Omega}(t) G\right|^{2} \leq T_{\Omega}(t)|G|^{2}, \quad t \geq 0, \text { v-a.e. in } \Omega \text {. }
$$

Moreover, taking into account that the semigroups $\mathbf{T}_{\Omega}(t)$ and $\mathbf{T}_{\varepsilon}(t)$ act component by component, we can obtain a vector-valued version of Theorem 1 .

Theorem 2. Under Hypotheses 1, 2 and 3, the following statements hold true.

(i) For any $\varepsilon>0$ and $F \in L^{2}\left(X, v_{\varepsilon} ; H\right)$, there exists a sequence $\left(F_{n}\right)_{n} \subseteq$ $L^{2}\left(X, v_{\varepsilon} ; H\right)$ such that $\mathbf{T}_{\varepsilon}(t) F_{n}$ belongs to $\mathcal{F} C_{b}^{3}(X ; H)$ and

$$
\begin{aligned}
& \lim _{n \rightarrow \infty}\left\|F_{n}-F\right\|_{L^{2}\left(X, v_{\varepsilon} ; H\right)}=0, \\
& \lim _{n \rightarrow \infty}\left\|\mathbf{T}_{\varepsilon}(t) F_{n}-\mathbf{T}_{\varepsilon}(t) F\right\|_{D^{1,2}\left(X, v_{\varepsilon} ; H\right)}=0, \quad t>0 .
\end{aligned}
$$

If, in addition, $F \in D^{1,2}\left(X, v_{\varepsilon} ; H\right)$ then $D_{H} F_{n}$ converges to $D_{H} F$ in $L^{1}\left(X, v_{\varepsilon} ; \mathcal{H}_{2}\right)$, as $n \rightarrow \infty$. 
(ii) For any $F \in L^{2}(\Omega, v ; H)$ there exists an infinitesimal sequence $\left(\varepsilon_{n}\right)_{n \in \mathbb{N}}$ such that $\mathbf{T}_{\varepsilon_{n}}(t) \widetilde{F}$ weakly converges to $\mathbf{T}_{\Omega}(t) F$ in $D^{1,2}(\Omega, v ; H)$, where $\widetilde{F}$ is any $L^{2}$-extension of $F$ to $X$.

Proof. (i) Let $F=\sum_{i=1}^{\infty} f^{(i)} e_{i}$ where $f^{(i)} \in L^{2}\left(X, v_{\varepsilon}\right), i \in \mathbb{N}$. For every $i \in \mathbb{N}$, by Theorem 1(i), there exists $\left(f_{k}^{(i)}\right)_{k \in \mathbb{N}} \subseteq L^{2}\left(X, v_{\varepsilon}\right)$ converging to $f^{(i)}$ in $L^{2}\left(X, v_{\varepsilon}\right)$ such that $T_{\varepsilon}(t) f_{k}^{(i)}$ belongs to $\mathcal{F} C_{b}^{3}(X)$ and

$$
\lim _{k \rightarrow \infty}\left\|T_{\varepsilon}(t) f_{k}^{(i)}-T_{\varepsilon}(t) f^{(i)}\right\|_{D^{1,2}\left(X, v_{\varepsilon}\right)}=0, \quad t, \varepsilon>0 .
$$

Observe that (12) follows immediately from (13). Now fix $i, n \in \mathbb{N}$ and consider $k_{i} \in \mathbb{N}$ such that for every $k \geq k_{i}$ it holds

$$
\int_{X}\left|f_{k}^{(i)}-f^{(i)}\right|^{2} \mathrm{~d} v_{\varepsilon}<\frac{1}{n 2^{i}}
$$

Consider the vector field $F_{n}:=\sum_{i=1}^{n} f_{k_{i}}^{(i)} e_{i}$. We claim that $\left(F_{n}\right)$ is the sequence we are looking for. Indeed $F_{n}$ belongs to $L^{2}\left(X, v_{\varepsilon} ; H\right)$ for any $n \in \mathbb{N}$. Let $n_{0} \in \mathbb{N}$ be such that $\sum_{i=n_{0}+1}^{\infty}\left\|f^{(i)}\right\|_{L^{2}\left(X, v_{\varepsilon}\right)}^{2} \leq \eta / 2$ and let $n \geq n_{0}$ such that $1 / n<\eta / 2$. We have

$$
\begin{aligned}
\left\|F_{n}-F\right\|_{L^{2}\left(X, v_{\varepsilon} ; H\right)}^{2} & \leq \sum_{i=1}^{n} \int_{X}\left|f_{k_{i}}^{(i)}-f^{(i)}\right|^{2} \mathrm{~d} v_{\varepsilon}+\sum_{i=n+1}^{\infty}\left\|f^{(i)}\right\|_{L^{2}\left(X, v_{\varepsilon}\right)}^{2} \\
& \leq \frac{1}{n}+\frac{\eta}{2} \leq \eta
\end{aligned}
$$

In a similar way we can prove the other statements.

(ii) is an immediate consequence of Proposition 4 and Theorem 1(ii).

Before going on, recall that usually in the characterisation of functions of bounded variation in terms of the short-time behaviour of suitable semigroups a crucial tool is an appropriate commutation formula between the semigroup and the gradient operator. For instance, for the Wiener space and the Ornstein-Uhlenbeck semigroup the equality $D_{H} S(t) f=e^{-t} \mathbf{S}(t) D_{H} f$ holds true for any $t \geq 0$. Let us prove a (quasi) commutation formula between $T_{\Omega}(t)$ and $D_{H}$, under the following additional assumption.

Hypotheses 4. The map $\left(d_{\Omega}\right)^{-2}\left\|D_{H}^{2} d_{\Omega}^{2}\right\|_{\mathcal{H}_{2}}$ belongs to $L^{2}(X, v)$.

Remark 3. It is not difficult to show that every open ball and every open ellipsoid of $X$ as well as every open hyperplane of $X$ satisfy Hypothesis 4 . We show that Hypothesis 4 is satisfied when $\Omega$ is the unit ball $B_{X}$ centred at zero. The other examples can be discussed in a similar fashion. Observe that, by Proposition 2, $\left\|D_{H}^{2} d_{\Omega}^{2}\right\|_{\mathcal{H}_{2}} \leq 2$ and $\left\|D_{H}^{2} d_{\Omega}^{2}(x)\right\|_{\mathcal{H}_{2}}=0$ if $x \in B_{X}$. Moreover, there exists a constant $C>0$ such that

$$
\begin{aligned}
d_{B_{X}}(x) & \geq C \inf \left\{|h|_{X} \mid h \in H \cap\left(B_{X}-x\right)\right\} \geq C \inf \left\{|h|_{X} \mid h \in\left(B_{X}-x\right)\right\} \\
& =C \inf \left\{|x-h|_{X} \mid h \in B_{X}\right\}=C \operatorname{dist}\left(x, B_{X}\right)=\left.C|| x\right|_{X}-1 \mid,
\end{aligned}
$$


where $\operatorname{dist}\left(x, B_{X}\right)$ is the distance of $x$ from $B_{X}$. So

$$
\begin{aligned}
& \int_{X} d_{B_{X}}^{-4}\left\|D_{H} d_{B_{X}}^{2}\right\|_{\mathcal{H}_{2}}^{2} \mathrm{~d} \nu \leq K \int_{X \backslash B_{X}} \frac{1}{\left(|x|_{X}-1\right)^{4}} \mathrm{~d} \nu(x) \\
& \quad \leq K \int_{X} \frac{1}{\left(|x|_{X}-1\right)^{4}} e^{-U(x)} \mathrm{d} \gamma(x) \leq K\left(\int_{X} e^{-p^{\prime} U} \mathrm{~d} \gamma\right)^{\frac{1}{p^{\prime}}}\left(\int_{X} \frac{1}{\left(|x|_{X}-1\right)^{4 p}} \mathrm{~d} \gamma(x)\right)^{\frac{1}{p}} \\
& \quad \leq K\left(\int_{X} e^{-p^{\prime} U} \mathrm{~d} \gamma\right)^{\frac{1}{p^{\prime}}}\left(\int_{\mathbb{R}^{n}} \frac{1}{\left(|\xi|_{\mathbb{R}^{n}}-1\right)^{4 p}} \mathrm{~d} \gamma_{n}(\xi)\right)^{\frac{1}{p}},
\end{aligned}
$$

where $K$ is a positive constant and $\gamma_{n}$ denotes the $n$-dimensional Gaussian measure, image of $\gamma$ under the projection on $\operatorname{span}\left\{v_{1}, \ldots, v_{n}\right\}$. To conclude, observe that there exists $n \in \mathbb{N}$ such that the right-hand side of (14) is finite.

Proposition 5. Under Hypotheses 1, 2, 3 and 4, the formula

$$
\begin{aligned}
& D_{H} T_{\Omega}(t) f-\left(e^{\left.-t Q_{\infty}^{-1} \mathbf{T}_{\Omega}(t) D_{H} f\right)}\right. \\
& \quad=-\int_{0}^{t} e^{(s-t) Q_{\infty}^{-1} \mathbf{T}_{\Omega}(t-s)\left(D_{H}^{2} U D_{H} T_{\Omega}(s) f\right) \mathrm{d} s .}
\end{aligned}
$$

holds true v-a.e. in $\Omega$, for any $f \in \operatorname{Lip}_{c}(\Omega)$ and $t>0$.

Proof. In order to prove (15) we show that

$$
\begin{aligned}
& \int_{\Omega}\left\langle D_{H} T_{\Omega}(t) f, G\right\rangle_{H} \mathrm{~d} v=\int_{\Omega}\left\langle e^{\left.-t Q_{\infty}^{-1} \mathbf{T}_{\Omega}(t) D_{H} f, G\right\rangle_{H} \mathrm{~d} v}\right. \\
& -\int_{\Omega} \int_{0}^{t}\left\langle e^{(s-t) Q_{\infty}^{-1}}\left(\mathbf{T}_{\Omega}(t-s)\left(D_{H}^{2} U D_{H} T_{\Omega}(s) f\right)\right), G\right\rangle_{H} \mathrm{~d} s \mathrm{~d} v,
\end{aligned}
$$

for any $f \in \operatorname{Lip}_{c}(\Omega), G \in C_{b}(\Omega ; H)$ and $t>0$. By performing slight changes in [12, Appendix A] we get

$$
\begin{aligned}
& D_{H} T_{\varepsilon}(t) g-\left(e^{\left.-t Q_{\infty}^{-1} \mathbf{T}_{\varepsilon}(t) D_{H} g\right)}\right. \\
& \quad=-\int_{0}^{t} e^{(s-t) Q_{\infty}^{-1} \mathbf{T}_{\varepsilon}(t-s)\left(D_{H}^{2} \Phi_{\varepsilon} D_{H} T_{\varepsilon}(s) g\right) \mathrm{d} s}
\end{aligned}
$$

$v_{\varepsilon}$-a.e. in $X$ for any $g \in \operatorname{Lip}_{b}(X)$ and $\varepsilon>0$, where $T_{\varepsilon}(t)$ is the semigroup introduced in Sect. 2.1. Now, let $f \in \operatorname{Lip}_{c}(\Omega)$ and $\tilde{f}$ be the trivial extension to zero of $f$ in the whole space $X$. Clearly, $\tilde{f}$ belongs to $\operatorname{Lip}_{b}(X)$ and (17) holds true with $g$ replaced by $\tilde{f}$. Consequently, multiplying (17) by the function $G$ and integrating on $\Omega$ with respect to $v$ yield

$$
\begin{aligned}
\int_{\Omega} & \left\langle D_{H} T_{\varepsilon}(t) \tilde{f}, G\right\rangle_{H} \mathrm{~d} v=\int_{\Omega}\left\langle e^{-t Q_{\infty}^{-1}}\left(\mathbf{T}_{\varepsilon}(t) D_{H} \tilde{f}\right), G\right\rangle_{H} \mathrm{~d} v \\
& -\int_{\Omega} \int_{0}^{t}\left\langle e^{(s-t) Q_{\infty}^{-1}}\left(\mathbf{T}_{\varepsilon}(t-s)\left(D_{H}^{2} \Phi_{\varepsilon} D_{H} T_{\varepsilon}(s) \widetilde{f}\right)\right), G\right\rangle_{H} \mathrm{~d} s \mathrm{~d} v \\
= & \int_{\Omega}\left\langle e^{\left.-t Q_{\infty}^{-1} \mathbf{T}_{\varepsilon}(t) D_{H} \tilde{f}, G\right\rangle_{H} \mathrm{~d} v}\right.
\end{aligned}
$$




$$
-\int_{0}^{t} \int_{\Omega}\left\langle e^{(s-t) Q_{\infty}^{-1}}\left(\mathbf{T}_{\varepsilon}(t-s)\left(D_{H}^{2} \Phi_{\varepsilon} D_{H} T_{\varepsilon}(s) \widetilde{f}\right)\right), G\right\rangle_{H} \mathrm{~d} \nu \mathrm{d} s,
$$

where in the last line we used the Fubini-Tonelli theorem.

The proof of (16) is split in two steps.

Step 1. We argue by approximation on the last terms in (18) and (16).

For every $\varepsilon, s>0$ we fix a Borel measurable version of $D_{H} T_{\Omega}(s) f$ and $D_{H} T_{\varepsilon}(s) \tilde{f}$ in $L^{2}(\Omega, v ; H)$ and $L^{2}\left(X, v_{\varepsilon} ; H\right)$, respectively. Consider the function

$$
\Gamma_{\varepsilon}(s, x):=\left\{\begin{array}{l}
D_{H} T_{\Omega}(s) f(x), x \in \Omega ; \\
D_{H} T_{\varepsilon}(s) \widetilde{f}(x), \quad x \in X \backslash \Omega .
\end{array}\right.
$$

Observe that the map $x \mapsto \Gamma_{\varepsilon}(s, x)$ is an extension of $D_{H} T_{\Omega}(s) f$ to the whole $X$. Thus, by Theorem 2 there is a sequence $\varepsilon_{n} \downarrow 0$ such that for every $\eta>0$ the function $\mathbf{T}_{\varepsilon_{n}}(t-s)\left(D_{H}^{2} U \Gamma_{\eta}(s, \cdot)\right)$ weakly converges to $\mathbf{T}_{\Omega}(t-s)\left(D_{H}^{2} U D_{H} T_{\Omega}(s) f\right)$ in $D^{1,2}(\Omega, v ; H)$. Observe that the set

$$
\left\{\mathbf{T}_{\varepsilon_{n}}(t-s)\left(D_{H}^{2} U \Gamma_{\eta}(s, \cdot)\right) \mid n \in \mathbb{N} \text { and } s, \eta>0\right\}
$$

is bounded in $L^{2}(\Omega, v ; H)$. Indeed by the contractivity of $\mathbf{T}_{\varepsilon_{n}}(t)$ in the space $L^{2}\left(X, v_{\varepsilon_{n}} ; H\right)$, the fact that $U \equiv \Phi_{\varepsilon}$ on $\Omega$ and estimate (8) we have

$$
\begin{aligned}
& \left\|\mathbf{T}_{\varepsilon_{n}}(t-s)\left(D_{H}^{2} U \Gamma_{\eta}(s, \cdot)\right)\right\|_{L^{2}(\Omega, v ; H)} \\
& \quad=\left\|\mathbf{T}_{\varepsilon_{n}}(t-s)\left(D_{H}^{2} U \Gamma_{\eta}(s, \cdot)\right)\right\|_{L^{2}\left(\Omega, v_{\varepsilon_{n}} ; H\right)} \\
& \quad \leq\left\|\mathbf{T}_{\varepsilon_{n}}(t-s)\left(D_{H}^{2} U \Gamma_{\eta}(s, \cdot)\right)\right\|_{L^{2}\left(X, v_{\varepsilon_{n}} ; H\right)} \\
& \quad \leq\left\|D_{H}^{2} U \Gamma_{\eta}(s, \cdot)\right\|_{L^{2}\left(X, v_{\varepsilon_{n}} ; H\right)} \leq\left[D_{H} U\right]_{H-\operatorname{Lip}}\left\|\Gamma_{\eta}(s, \cdot)\right\|_{L^{2}\left(X, v_{\varepsilon_{n}} ; H\right)} \\
& \quad \leq\left[D_{H} U\right]_{H \text {-Lip }}\left(\left\|D_{H} T_{\eta}(s) \widetilde{f}\right\|_{L^{2}\left(X, v_{\varepsilon_{n}} ; H\right)}+\left\|D_{H} T_{\Omega}(s) f\right\|_{L^{2}(\Omega, v ; H)}\right) \\
& \quad \leq\left[D_{H} U\right]_{H-\operatorname{Lip}} e^{-2 \lambda_{1}^{-1} s}\left(\left\|T_{\eta}(s)\left|D_{H} \widetilde{f}\right|_{H}\right\|_{L^{2}\left(X, v_{\varepsilon_{n}}\right)}+\left\|T_{\Omega}(s)\left|D_{H} f\right|_{H}\right\|_{L^{2}(\Omega, v)}\right) \\
& \quad \leq 2\left[D_{H} U\right]_{H-\operatorname{Lip}}(v(X))^{\frac{1}{2}}\left\|D_{H} f\right\|_{L^{\infty}(\Omega, v ; H)},
\end{aligned}
$$

where in the last line we used the contractivity of $T_{\eta}(t)$ and $T_{\Omega}(t)$ in $L^{\infty}$ and the fact that $v_{\varepsilon_{n}}(X) \leq v(X)$ for any $n \in \mathbb{N}$. So there exists $M>0$ large enough so that the family in (19) is contained in $B(0, M)$, the ball of $L^{2}(\Omega, v ; H)$ with centre 0 and radius $M$.

Recall that every bounded subset of $L^{2}(\Omega, v ; H)$ is weakly metrisable (see [15, Proposition 3.106]) and let $\rho: B(0, M) \times B(0, M) \rightarrow \mathbb{R}$ be a metric such that the topology generated by $\rho$ and the weak topology in $B(0, M)$ coincide. Now we use a diagonal argument to pass to the limit in (18). Let $n_{1} \in \mathbb{N}$ be such that for every $n \geq n_{1}$ it holds

$$
\rho\left(\mathbf{T}_{\varepsilon_{n}}(t-s)\left(D_{H}^{2} U \bar{\Gamma}_{1}(s, \cdot)\right), \mathbf{T}_{\Omega}(t-s)\left(D_{H}^{2} U D_{H} T_{\Omega}(s) f\right)\right) \leq 1,
$$


where $\bar{\Gamma}_{j}(s, x)=\Gamma_{j^{-1}}(s, x)$ for any $s>0$ and $x \in X$. Now assume that $n_{1}, \ldots, n_{k}$ are already constructed and consider $n_{k+1}>n_{k}$ such that for every $n \geq n_{k+1}$

$$
\rho\left(\mathbf{T}_{\varepsilon_{n}}(t-s)\left(D_{H}^{2} U \bar{\Gamma}_{n_{k}}(s, \cdot)\right), \mathbf{T}_{\Omega}(t-s)\left(D_{H}^{2} U D_{H} T_{\Omega}(s) f\right)\right) \leq \frac{1}{2^{k}} .
$$

Consider now the sequence $\left(\mathbf{T}_{\varepsilon_{n_{k}}}(t-s)\left(D_{H}^{2} U \bar{\Gamma}_{n_{k}}(s, \cdot)\right)\right)_{k \in \mathbb{N}}$ and observe that it weakly converges to $\mathbf{T}_{\Omega}(t-s)\left(D_{H}^{2} U D_{H} T_{\Omega}(s) f\right)$ in $L^{2}(\Omega, v ; H)$ as $k \rightarrow \infty$.

Step 2. To complete the proof, we replace $\varepsilon$ in (18) by a sequence $\varepsilon_{m} \downarrow 0$ such that step 1 and Theorems 1, 2 apply. Let us show that we can take the limit as $m \rightarrow \infty$. Indeed, from Theorem 1 it follows that for any $f \in L^{2}(\Omega, v), T_{\varepsilon_{m}}(t) \widetilde{f}$ weakly converges (up to a subsequence) to $T_{\Omega}(t) f$ in $D^{2,2}(\Omega, v)$ as $m \rightarrow \infty$, hence writing $T_{m}, \mathbf{T}_{m}, \Phi_{m}, \Gamma_{m}$ in place of $T_{\varepsilon_{m}}, \mathbf{T}_{\varepsilon_{m}}, \Phi_{\varepsilon_{m}}, \Gamma_{\varepsilon_{m}}$ we obtain

$$
\lim _{m \rightarrow \infty} \int_{\Omega}\left\langle D_{H} T_{m}(t) \tilde{f}, G\right\rangle_{H} \mathrm{~d} v=\int_{\Omega}\left\langle D_{H} T_{\Omega}(t) f, G\right\rangle_{H} \mathrm{~d} v
$$

and by the analogous vector-valued result (see Theorem 2, (10) and again (7))

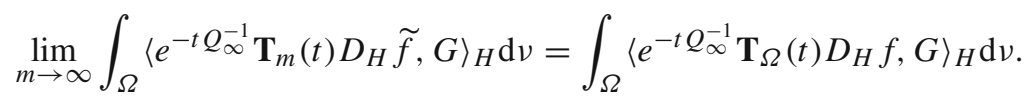

To conclude we have to prove that the last term in the right-hand side of (18) converges to the last term in the right-hand side of (16).

$$
\begin{aligned}
& \left|\int_{\Omega}\left\langle\mathbf{T}_{m}(t-s)\left(D_{H}^{2} \Phi_{m} D_{H} T_{m}(s) \tilde{f}\right) \mathrm{d} v-\int_{\Omega} \mathbf{T}_{\Omega}(t-s)\left(D_{H}^{2} U D_{H} T_{\Omega}(s) f\right), G\right\rangle_{H} \mathrm{~d} v\right| \\
& \leq\left|\int_{\Omega}\left\langle\mathbf{T}_{m}(t-s)\left(D_{H}^{2} \Phi_{m} D_{H} T_{m}(s) \tilde{f}\right)-\mathbf{T}_{m}(t-s)\left(D_{H}^{2} \Phi_{m} \Gamma_{m}(s, \cdot)\right), G\right\rangle_{H} \mathrm{~d} v\right| \\
& \quad+\left|\int_{\Omega}\left\langle\mathbf{T}_{m}(t-s)\left(D_{H}^{2} \Phi_{m} \Gamma_{m}(s, \cdot)\right)-\mathbf{T}_{m}(t-s)\left(D_{H}^{2} U \Gamma_{m}(s, \cdot)\right), G\right\rangle_{H} \mathrm{~d} v\right| \\
& \quad+\left|\int_{\Omega}\left\langle\mathbf{T}_{m}(t-s)\left(D_{H}^{2} U \Gamma_{m}(s, \cdot)\right)-\mathbf{T}_{\Omega}(t-s)\left(D_{H}^{2} U D_{H} T_{\Omega}(s) f\right), G\right\rangle_{H} \mathrm{~d} v\right| \\
& =: I_{1}(m)+I_{2}(m)+I_{3}(m) .
\end{aligned}
$$

Let us estimate $I_{1}$. Using that $\Phi_{m} \equiv U$ on $\Omega$ for every $m \in \mathbb{N}$, formula (10) and the invariance property of $T_{m}$ with respect to $v_{m}:=v_{\varepsilon_{m}}$ we have

$$
\begin{aligned}
& I_{1}(m) \leq \int_{\Omega} \mid \mathbf{T}_{m}(t-s)\left(D_{H}^{2} \Phi_{m} D_{H} T_{m}(s) \tilde{f}\right) \\
& \quad-\left.\mathbf{T}_{m}(t-s)\left(D_{H}^{2} \Phi_{m} \Gamma_{m}(s, \cdot)\right)\right|_{H}|G|_{H} \mathrm{~d} v \\
& \leq(v(X))^{\frac{1}{2}}\|G\|_{\infty}\left(\int_{\Omega}\left|\mathbf{T}_{m}(t-s)\left(D_{H}^{2} \Phi_{m} D_{H} T_{m}(s) \tilde{f}-D_{H}^{2} \Phi_{m} \Gamma_{m}(s, \cdot)\right)\right|_{H}^{2} \mathrm{~d} v\right)^{\frac{1}{2}} \\
& \leq(v(X))^{\frac{1}{2}}\|G\|_{\infty}\left(\int_{\Omega} T_{m}(t-s)\left|D_{H}^{2} \Phi_{m} D_{H} T_{m}(s) \tilde{f}-D_{H}^{2} \Phi_{m} \Gamma_{m}(s, \cdot)\right|_{H}^{2} \mathrm{~d} v_{m}\right)^{\frac{1}{2}}
\end{aligned}
$$




$$
\begin{aligned}
& \leq(v(X))^{\frac{1}{2}}\|G\|_{\infty}\left(\int_{X} T_{m}(t-s)\left|D_{H}^{2} \Phi_{m} D_{H} T_{m}(s) \tilde{f}-D_{H}^{2} \Phi_{m} \Gamma_{m}(s, \cdot)\right|_{H}^{2} \mathrm{~d} v_{m}\right)^{\frac{1}{2}} \\
& =(v(X))^{\frac{1}{2}}\|G\|_{\infty}\left(\int_{X}\left|\left(D_{H}^{2} \Phi_{m} D_{H} T_{m}(s) \tilde{f}\right)-\left(D_{H}^{2} \Phi_{m} \Gamma_{m}(s, \cdot)\right)\right|_{H}^{2} \mathrm{~d} v_{m}\right)^{\frac{1}{2}} \\
& \leq(v(X))^{\frac{1}{2}}\|G\|_{\infty}\left(\int_{\Omega}\left\|D_{H}^{2} U\right\|_{\mathcal{H}_{2}}^{2}\left|D_{H} T_{m}(s) \tilde{f}-D_{H} T_{\Omega}(s) f\right|_{H}^{2} \mathrm{~d} v\right)^{\frac{1}{2}} \\
& \leq\left[D_{H} U\right]_{H-\operatorname{Lip}}(v(X))^{\frac{1}{2}}\|G\|_{\infty}\left(\int_{\Omega}\left|D_{H} T_{m}(s) \tilde{f}-D_{H} T_{\Omega}(s) f\right|_{H}^{2} \mathrm{~d} v\right)^{\frac{1}{2}} \cdot
\end{aligned}
$$

The right-hand side of (20) converges to zero as $m \rightarrow \infty$ : indeed, $D_{H} T_{m}(s) \tilde{f}$ converges pointwise $\nu$-almost everywhere in $\Omega$ to $D_{H} T_{\Omega}(s) f$. Furthermore, by Proposition 3 we have that $v$-a.e. in $\Omega$

$$
\begin{aligned}
& \left|D_{H} T_{\varepsilon_{m}}(s) \tilde{f}-D_{H} T_{\Omega}(s) f\right|_{H}^{2} \leq 2\left(\left|D_{H} T_{\varepsilon_{m}}(s) \tilde{f}\right|_{H}^{2}+\left|D_{H} T_{\Omega}(s) f\right|_{H}^{2}\right) \\
& \leq 2 e^{-2 \lambda_{1}^{-1} s}\left(T_{\varepsilon_{m}}(s)\left|D_{H} \tilde{f}\right|_{H}^{2}+T_{\Omega}(s)\left|D_{H} f\right|_{H}^{2}\right) \leq 2\left\|D_{H} f\right\|_{L^{\infty}(\Omega, v ; H)}
\end{aligned}
$$

So by the dominated convergence theorem we get that $I_{1}(m)$ vanishes as $m \rightarrow \infty$. Now, using similar arguments we can estimate $I_{2}(m)$ as follows

$$
\begin{aligned}
I_{2}(m) \leq & (v(X))^{\frac{1}{2}}\|G\|_{\infty}\left(\int_{X}\left|\left(D_{H}^{2} \Phi_{m} \Gamma_{m}(s, \cdot)\right)-\left(D_{H}^{2} U \Gamma_{m}(s, \cdot)\right)\right|_{H}^{2} \mathrm{~d} v_{m}\right)^{\frac{1}{2}} \\
\leq & (v(X))^{\frac{1}{2}}\|G\|_{\infty}\left(\int_{X}\left\|D_{H}^{2} \Phi_{m}-D_{H}^{2} U\right\|_{\mathcal{H}_{2}}^{2}\left|\Gamma_{m}(s, \cdot)\right|_{H}^{2} \mathrm{~d} v_{m}\right)^{\frac{1}{2}} \\
\leq & (v(X))^{\frac{1}{2}}\|G\|_{\infty}\left(\int_{X}\left\|D_{H}^{2} \Phi_{m}-D_{H}^{2} U\right\|_{\mathcal{H}_{2}}^{2} \mid\left(D_{H} T_{m}(s) \widetilde{f}\right) \chi_{X \backslash \Omega}\right. \\
& \left.+\left.\left(D_{H} T_{\Omega}(s) f\right) \chi_{\Omega}\right|_{H} ^{2} \mathrm{~d} v_{m}\right)^{\frac{1}{2}} \\
\leq & (2 v(X))^{\frac{1}{2}}\|G\|_{\infty}\left(\int _ { X } \| D _ { H } ^ { 2 } \Phi _ { m } - D _ { H } ^ { 2 } U \| _ { \mathcal { H } _ { 2 } } ^ { 2 } \left(\left|D_{H} T_{m}(s) \widetilde{f}\right|_{H}^{2}\right.\right. \\
& \left.\left.+\left|D_{H} T_{\Omega}(s) f\right|_{H}^{2} \chi_{\Omega}\right) \mathrm{d} v_{m}\right)^{\frac{1}{2}} \\
\leq & (2 v(X))^{\frac{1}{2}}\|G\|_{\infty}\left(\int _ { X } \| D _ { H } ^ { 2 } \Phi _ { m } - D _ { H } ^ { 2 } U \| _ { \mathcal { H } _ { 2 } } ^ { 2 } \left(T_{m}(s)\left|D_{H} \widetilde{f}\right|_{H}^{2}\right.\right. \\
& \left.\left.+\left(T_{\Omega}(s)\left|D_{H} f\right|_{H}^{2}\right) \chi_{\Omega}\right) \mathrm{d} v_{m}\right)^{\frac{1}{2}} \\
\leq & 2(v(X))^{\frac{1}{2}}\left\|D_{H} f\right\|_{L^{\infty}(\Omega, v ; H)}\|G\|_{\infty}\left(\int_{X}\left\|D_{H}^{2} \Phi_{m}-D_{H}^{2} U\right\|_{\mathcal{H}}^{2} \mathrm{~d} v_{m}\right)^{\frac{1}{2}} \\
= & 2(v(X))^{\frac{1}{2}}\left\|D_{H} f\right\|_{L^{\infty}(\Omega, v ; H)}\|G\|_{\infty}\left(\int_{X} \frac{1}{4 \varepsilon_{m}}\left\|D_{H}^{2} d_{\Omega}^{2}\right\|_{\mathcal{H}_{2}}^{2} e^{-U-\frac{1}{2 \varepsilon_{m}} d_{\Omega}^{2}} \mathrm{~d} \gamma\right)^{\frac{1}{2}} .
\end{aligned}
$$


Now observe that the right-hand side of (21) vanishes as $m \rightarrow \infty$. Indeed the function $\frac{1}{4 \varepsilon_{m}}\left\|D_{H}^{2} d_{\Omega}^{2}\right\|_{\mathcal{H}_{2}}^{2} e^{-U-\frac{1}{2 \varepsilon_{m}} d_{\Omega}^{2}}$ identically vanishes in $\Omega$ and converges pointwise to 0 $\nu$-almost everywhere in $X \backslash \Omega$ as $m \rightarrow \infty$. Furthermore, observe that the function $(0, \infty) \ni \varepsilon \mapsto R(\varepsilon):=\frac{1}{4 \varepsilon^{2}}\left|D_{H}^{2} d_{\Omega}^{2}\right|^{2} e^{-\frac{1}{2 \varepsilon} d_{\Omega}^{2}}$ attains its maximum in $\varepsilon=d_{\Omega}^{2} / 4$ where it equals to $4 d_{\Omega}^{-4} \|\left. D_{H}^{2} d_{\Omega}^{2}\right|_{\mathcal{H}_{2}} ^{2}$. Thus, using Hypothesis 4 and applying the dominated convergence theorem we infer that also $I_{2}(m)$ converges to zero as $m$ goes to infinity.

Finally $I_{3}(m)$ converges to zero as $m$ goes to infinity thanks to step 1 and this concludes the proof.

Corollary 1. Assume Hypotheses 1, 2, 3 and 4 hold true. For any $t>0$ and $p>1$ there exist two operators $\mathbf{S}_{1}(t): L^{p}(\Omega, v ; H) \rightarrow L^{1}(\Omega, v ; H)$ and $S_{2}(t): L^{p}(\Omega, v) \rightarrow$ $L^{1}(\Omega, v ; H)$ such that for every continuous and $H$-differentiable function $\varphi: \Omega \rightarrow \mathbb{R}$ with H-Lipschitz gradient

$$
D_{H} T_{\Omega}(t) \varphi=\mathbf{S}_{1}(t) D_{H} \varphi+S_{2}(t) \varphi \text {. }
$$

Moreover, the adjoint operator $\left(\mathbf{S}_{1}(t)\right)^{*}$ maps $\operatorname{Lip}_{c}(\Omega ; H)$ into $L^{\infty}(\Omega, v ; H)$ and verifies $\left\|\mathbf{S}_{1}^{*}(t) F\right\|_{\infty} \leq C_{1}(t)\|F\|_{\infty}$ for any $F \in \operatorname{Lip}_{c}(\Omega ; H)$ with $C_{1}(t) \rightarrow 1$ as $t \rightarrow 0$ and the norm $C_{2}(t):=\left\|S_{2}(t)\right\|_{\mathcal{L}\left(L^{p}, L^{1}\right)} \rightarrow 0$ as $t \rightarrow 0$.

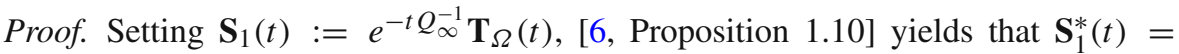

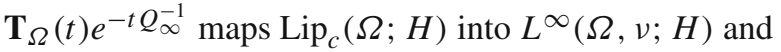

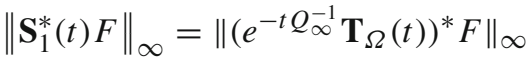

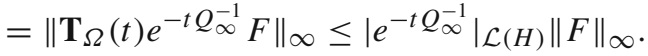

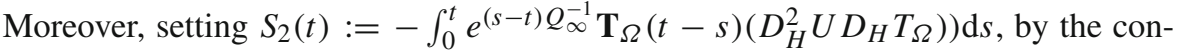
tractivity of $\mathbf{T}_{\Omega}(t)$ in $L^{1}(\Omega, v ; H),(7)$, Hypothesis 2 , the contractivity of $e^{-t Q_{\infty}^{-1}}$ in $H$, estimate (11) and the invariance property of $T_{\Omega}(t)$, we get

$$
\begin{aligned}
& \left\|\mathbf{S}_{2}(t) \varphi\right\|_{L^{1}(\Omega, v ; H)}
\end{aligned}
$$

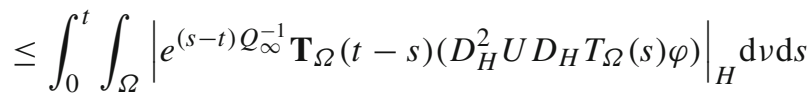

$$
\begin{aligned}
& \leq \int_{0}^{t}\left|e^{(s-t) Q_{\infty}^{-1}}\right|_{\mathcal{L}(H)} \int_{\Omega}\left|\mathbf{T}_{\Omega}(t-s)\left(D_{H}^{2} U D_{H} T_{\Omega}(s) \varphi\right)\right|_{H} \mathrm{~d} \nu \mathrm{d} s \\
& \leq \int_{0}^{t} \int_{\Omega}\left|\mathbf{T}_{\Omega}(t-s)\left(D_{H}^{2} U D_{H} T_{\Omega}(s) \varphi\right)\right|_{H} \mathrm{~d} \nu \mathrm{d} s \\
& \leq \int_{0}^{t} \int_{\Omega}\left|D_{H}^{2} U D_{H} T_{\Omega}(s) \varphi\right|_{H} \mathrm{~d} \nu \mathrm{d} s \\
& \leq \int_{0}^{t} \int_{\Omega}\left\|D_{H}^{2} U\right\|_{\mathcal{H}_{2}}\left|D_{H} T_{\Omega}(s) \varphi\right|_{H} \mathrm{~d} v \mathrm{~d} s \\
& \leq \int_{0}^{t}\left(\int_{\Omega}\left\|D_{H}^{2} U\right\|_{\mathcal{H}_{2}}^{p^{\prime}} \mathrm{d} v\right)^{\frac{1}{p^{\prime}}}\left(\int_{\Omega}\left|D_{H} T_{\Omega}(s) \varphi\right|_{H}^{p} \mathrm{~d} v\right)^{\frac{1}{p}} \mathrm{~d} s
\end{aligned}
$$




$$
\begin{aligned}
& \leq K_{p}^{\frac{1}{p}}\left\|D_{H}^{2} U\right\|_{L^{p^{\prime}}\left(X, v ; \mathcal{H}_{2}\right)} \int_{0}^{t} s^{-\frac{1}{2}}\left(\int_{\Omega} T_{\Omega}(s)|\varphi|^{p} \mathrm{~d} v\right)^{\frac{1}{p}} \mathrm{~d} s \\
& \leq K_{p}^{\frac{1}{p}}\left\|D_{H}^{2} U\right\|_{L^{p^{\prime}}\left(X, v ; \mathcal{H}_{2}\right)}\|\varphi\|_{L^{p}(\Omega, v)} \int_{0}^{t} s^{-\frac{1}{2}} \mathrm{~d} s \\
& =2 K_{p}^{\frac{1}{p}} \sqrt{t}\left\|D_{H}^{2} U\right\|_{L^{p^{\prime}}\left(X, v ; \mathcal{H}_{2}\right)}\|\varphi\|_{L^{p}(\Omega, v)}
\end{aligned}
$$

for any $t>0$. By the assumption on $U$ we deduce that the operator $S_{2}(t)$ is bounded from $L^{p}(\Omega, v)$ into $L^{1}(\Omega, v ; H)$ for any $t>0$. Finally, estimates (22) and (23) allow us to complete the proof.

Now, we are able to prove the main result of this section.

Theorem 3. Assume Hypotheses 1, 2, 3 and 4 hold true and let $u \in L^{2}(\Omega, v)$. The following statements are true:

(i) if $\liminf _{t \rightarrow 0^{+}}\left\|D_{H} T_{\Omega}(t) u\right\|_{L^{1}(\Omega, v ; H)}$ is finite, then $u \in B V(\Omega, v)$;

(ii) if $u \in B V(\Omega, v)$, then $\lim \sup _{t \rightarrow 0^{+}}\left\|D_{H} T_{\Omega}(t) u\right\|_{L^{1}(\Omega, v ; H)} \leq\left|D_{v} u\right|(\Omega)$.

Hence, $u \in B V(\Omega, v)$ iff $\lim _{t \rightarrow 0^{+}}\left\|D_{H} T_{\Omega}(t) u\right\|_{L^{1}(\Omega, v ; H)}<\infty$. In this case

$$
\left|D_{\nu} u\right|(\Omega)=\lim _{t \rightarrow 0^{+}}\left\|D_{H} T_{\Omega}(t) u\right\|_{L^{1}(\Omega, \nu ; H)} .
$$

Proof. (i) follows from the strong continuity of $T_{\Omega}(t)$ in $L^{1}(\Omega, v)$, see Proposition 3(i), and the lower semicontinuity of the norm (9), which imply

$$
\left|D_{\nu} u\right|(\Omega) \leq \liminf _{t \rightarrow 0^{+}} \int_{\Omega}\left|D_{H} T_{\Omega}(t) u\right|_{H} \mathrm{~d} \nu
$$

To prove (ii) we write the $L^{1}$-norm of the gradient of $T_{\Omega}(t) u$ by duality, as

$$
\left\|D_{H} T_{\Omega}(t) u\right\|_{L^{1}(\Omega, v ; H)}=\sup \left\{\begin{array}{l|l}
\int_{\Omega}\left\langle D_{H} T_{\Omega}(t) u, F\right\rangle_{H} \mathrm{~d} v & \begin{array}{l}
F \in \operatorname{Lip}_{c}(\Omega ; H), \\
\|F\|_{\infty} \leq 1
\end{array}
\end{array}\right\} .
$$

Taking into account that, for any $F \in \operatorname{Lip}_{b}(\Omega ; H)$ we get

$$
\begin{aligned}
& \int_{\Omega}\left\langle D_{H} T_{\Omega}(t) u, F\right\rangle_{H} \mathrm{~d} v=\int_{\Omega} u\left(D_{H} T_{\Omega}(t)\right)^{*} F \mathrm{~d} v \\
& \quad \leq \int_{\Omega} u\left(\mathbf{S}_{1}(t) D_{H}+S_{2}(t)\right)^{*} F \mathrm{~d} v=\int_{\Omega} u\left(D_{H}^{*} \mathbf{S}_{1}(t)^{*} F+S_{2}(t)^{*} F\right) \mathrm{d} v \\
& \quad \leq\left|D_{v} u\right|(\Omega)\left\|\mathbf{S}_{1}(t)^{*} F\right\|_{\infty}+\|u\|_{L^{2}(\Omega, v)}\left\|S_{2}(t)^{*} F\right\|_{L^{2}(\Omega, v)} \\
& \quad \leq\left(\left|D_{v} u\right|(\Omega) C_{1}(t)+C_{2}(t)\right)\|F\|_{\infty}
\end{aligned}
$$

we deduce that

$$
\left\|D_{H} T_{\Omega}(t) u\right\|_{L^{1}(\Omega, v ; H)} \leq C_{1}(t)\left|D_{\nu} u\right|(\Omega)+C_{2}(t)
$$


for any $t>0$ where $C_{i}(i=1,2)$ are the positive functions in Corollary 1 . Thus, taking the limsup as $t \rightarrow 0^{+}$in (25) we get

$$
\limsup _{t \rightarrow 0^{+}} \int_{\Omega}\left|D_{H} T_{\Omega}(t) u\right|_{H} \mathrm{~d} \nu \leq\left|D_{\nu} u\right|(\Omega)
$$

and the proof is complete.

It follows from Theorem 3 that functions in $B V(\Omega, v)$ may be approximated in variation by smooth functions. This result was already known in infinite dimension when $\Omega=X$ and $T_{\Omega}(t)$ is the Ornstein-Uhlenbeck semigroup and in a convex set, see [21], where the approximation is based on finite-dimensional reductions of the semigroup generated by the Neumann Ornstein-Uhlenbeck operator in $\Omega$.

Proposition 6. Under Hypotheses 1, 2, 3 and 4, for any $f \in B V(\Omega, v)$ there exists a sequence $\left(f_{n}\right)_{n \in \mathbb{N}} \subseteq D^{1,2}(\Omega, v)$ such that

(i) $\lim _{n \rightarrow \infty}\left\|f_{n}-f\right\|_{L^{2}(\Omega, v)}=0$ and (ii) $\lim _{n \rightarrow \infty} \int_{\Omega}\left|D_{H} f_{n}\right|_{H} \mathrm{~d} v=\left|D_{\nu} f\right|(\Omega)$.

If $C \subseteq \Omega$ is closed and $\left|D_{v} f\right|(\partial C)=0$ then $\left|D_{v} f\right|(C)=\lim _{n \rightarrow \infty} \int_{C}\left|D_{H} f_{n}\right|_{H} \mathrm{~d} v$.

Proof. Consider the semigroup $T_{\Omega}(t)$ generated in $L^{2}(\Omega, v)$ by the operator $L_{\Omega}$ defined in (4). It is known that for any $f \in L^{2}(\Omega, v)$ the function $T_{\Omega}(t) f$ belongs to $D^{1,2}(\Omega, v)$ for any $t>0$ and by the strong continuity of $T_{\Omega}(t), T_{\Omega}(t) f$ converges to $f$ in $L^{2}(\Omega, v)$ as $t \rightarrow 0^{+}$. Moreover, Theorem 3 implies that $\left\|D_{H} T_{\Omega}(t) f\right\|_{L^{1}(\Omega, v ; H)}$ converges to $\left|D_{v} f\right|(\Omega)$ as $t \rightarrow 0^{+}$. Thus, (26) is proved. To complete the proof let us observe that, by the lower semicontinuity of the total variation, for any open set $A \subseteq \Omega$

$$
\left|D_{v} f\right|(A) \leq \liminf _{n \rightarrow \infty} \int_{A}\left|D_{H} f_{n}\right|_{H} \mathrm{~d} v
$$

(see [21, Corollary 2.5]). Analogously we deduce that

$$
\left|D_{\nu} f\right|(C) \geq \limsup _{n \rightarrow \infty} \int_{C}\left|D_{H} f_{n}\right|_{H} \mathrm{~d} v
$$

for any closed subset $C \subseteq \Omega$. Indeed, by (27) we obtain

$$
\begin{aligned}
& \left|D_{v} f\right|(\Omega)-\left|D_{v} f\right|(C)=\left|D_{\nu} f\right|(\Omega \backslash C) \leq \liminf _{n \rightarrow \infty} \int_{\Omega \backslash C}\left|D_{H} f_{n}\right|_{H} \mathrm{~d} v \\
& =\liminf _{n \rightarrow \infty}\left(\int_{\Omega}\left|D_{H} f_{n}\right|_{H} \mathrm{~d} v-\int_{C}\left|D_{H} f_{n}\right|_{H} \mathrm{~d} v\right) \\
& =\lim _{n \rightarrow \infty} \int_{\Omega}\left|D_{H} f_{n}\right|_{H} \mathrm{~d} v-\limsup _{n \rightarrow \infty} \int_{C}\left|D_{H} f_{n}\right|_{H} \mathrm{~d} v
\end{aligned}
$$


whence, using (26)(ii), estimate (28) follows. Now, using estimates (27), (28) and the fact that $\left|D_{v} f\right|(\partial C)=0$ we obtain

$$
\begin{aligned}
\left|D_{\nu} f\right|(C)=\left|D_{\nu} f\right|(\stackrel{\circ}{C}) & \leq \liminf _{n \rightarrow \infty} \int_{\check{C}}\left|D_{H} f_{n}\right|_{H} \mathrm{~d} v \\
& \leq \limsup _{n \rightarrow \infty} \int_{\check{C}}\left|D_{H} f_{n}\right|_{H} \mathrm{~d} v \leq\left|D_{\nu} f\right|(C),
\end{aligned}
$$

where $\stackrel{\circ}{C}$ denotes the interior of $C$. Estimate (29) yields the claim.

We conclude this section showing that estimate (8) and the previous approximation result allow to improve estimate (25) obtaining (30).

Theorem 4. Under Hypotheses 1, 2, 3 and 4, if $f \in B V(\Omega, v)$ then

$$
\int_{\Omega}\left|D_{H} T_{\Omega}(t) f\right|_{H} \mathrm{~d} v \leq e^{-\lambda_{1}^{-1} t}\left|D_{\nu} f\right|(\Omega), \quad t>0,
$$

$\lambda_{1}$ being the maximum eigenvalue of the covariance operator $Q_{\infty}$, see (2). Moreover, for any open set $A \subset \Omega$ with $\bar{A} \subset \Omega$,

$$
\lim _{t \rightarrow 0^{+}} \int_{A}\left|D_{H} T_{\Omega}(t) f\right|_{H} \mathrm{~d} v=\left|D_{v} f\right|(A) .
$$

Proof. Let $f \in B V(\Omega, v)$ and let $\left(f_{n}\right)_{n \in \mathbb{N}} \in D^{1,2}(\Omega, v)$ be the sequence given by Proposition 6. By the contractivity of $T_{\Omega}(t)$ we deduce that $T_{\Omega}(t) f_{n}$ converges to $T_{\Omega}(t) f$ in $L^{2}(\Omega, v)$ as $n \rightarrow \infty$. This fact, together with the lower semicontinuity of the total variation, (8) and (6) yield

$$
\begin{aligned}
& \int_{\Omega}\left|D_{H} T_{\Omega}(t) f\right|_{H} \mathrm{~d} v \leq \liminf _{n \rightarrow \infty} \int_{\Omega}\left|D_{H} T_{\Omega}(t) f_{n}\right|_{H} \mathrm{~d} v \\
& \quad \leq e^{-\lambda_{1}^{-1} t} \liminf _{n \rightarrow \infty} \int_{\Omega} T_{\Omega}(t)\left|D_{H} f_{n}\right|_{H} \mathrm{~d} v \leq e^{-\lambda_{1}^{-1} t} \lim _{n \rightarrow \infty} \int_{\Omega}\left|D_{H} f_{n}\right|_{H} \mathrm{~d} v \\
& =e^{-\lambda_{1}^{-1} t}\left|D_{\nu} f\right|(\Omega)
\end{aligned}
$$

whence (30) is proved. The last assertion follows immediately from Proposition 6 taking into account that $T_{\Omega}(t) f$ satisfies (26).

\section{Sets of finite perimeter in $\Omega$}

This section is devoted to provide some sufficient and necessary conditions in order that a Borel set $E \subseteq X$ have finite perimeter in $\Omega$. We consider also the case of $B V(\Omega, v)$ functions and $\Omega=X$. There are three semigroups involved: beside $T_{\Omega}(t)$, we consider the Ornstein-Uhlenbeck semigroup $S(t)$ generated in $L^{2}(X, \gamma)$ by the realisation of the operator

$$
L_{O U} \varphi=\operatorname{Tr}\left(D_{H}^{2} \varphi\right)-\sum_{i=1}^{\infty} \lambda_{i}^{-1}\left\langle x, e_{i}\right\rangle D_{i} \varphi \quad \varphi \in \mathcal{F} C_{b}^{2}(X)
$$


and the semigroup $T(t)$ generated in $L^{2}(X, v)$ by the realisation of the operator

$$
L \varphi=L_{O U} \varphi-\left\langle D_{H} U, D_{H} \varphi\right\rangle_{H}, \quad \varphi \in \mathcal{F} C_{b}^{2}(X) .
$$

Recall that $S(t)$ admits a pointwise representation by means of the Mehler formula (1).

Theorem 5. Assume Hypotheses 1, 2, 3 and 4 hold true and let $E \subseteq X$ be a Borel set such that $P_{v}(E, \Omega)<\infty$. Then

$$
\limsup _{t \rightarrow 0^{+}} \frac{1}{\sqrt{t}}\left\|T_{\Omega}(t) \chi_{E}-\chi_{E}\right\|_{L^{1}(\Omega, v)}<\infty .
$$

More precisely

$$
\left\|T_{\Omega}(t) u-u\right\|_{L^{1}(\Omega, v)} \leq 2 \sqrt{K_{2} t}\left|D_{\nu} u\right|(\Omega)
$$

for any $u \in B V(\Omega, v)$ and $t>0$ where $K_{2}$ is the constant in (7).

Proof. Clearly, once estimate (33) is proved, (32) follows at once choosing $u=\chi_{E}$. Thus, let us prove (33). To this aim, we consider $g \in L^{\infty}(\Omega, v)$ and assume first that $u \in D^{1,2}(\Omega, v)$. By the self-adjointness of the operators $L_{\Omega} T_{\Omega}(s)$ for $s \geq 0$ in $L^{2}(\Omega, v)$ we have

$$
\begin{gathered}
\int_{\Omega} g\left(T_{\Omega}(t) u-u\right) \mathrm{d} v=\int_{\Omega} g \int_{0}^{t} \frac{\mathrm{d}}{\mathrm{d} s} T_{\Omega}(s) u \mathrm{~d} s \mathrm{~d} \nu=\int_{0}^{t} \int_{\Omega} g\left(L_{\Omega} T_{\Omega}(s) u\right) \mathrm{d} \nu \mathrm{d} s \\
=\int_{0}^{t} \int_{\Omega}\left(L_{\Omega} T_{\Omega}(s) g\right) u \mathrm{~d} \nu \mathrm{d} s=-\int_{0}^{t} \int_{\Omega}\left\langle D_{H} T_{\Omega}(s) g, D_{H} u\right\rangle_{H} \mathrm{~d} \nu \mathrm{d} s .
\end{gathered}
$$

The Cauchy-Schwarz inequality and (7) yield

$$
\begin{aligned}
\int_{\Omega} g\left(T_{\Omega}(t) u-u\right) \mathrm{d} v & \leq \int_{0}^{t} \int_{\Omega}\left|D_{H} T_{\Omega}(s) g\right|_{H}\left|D_{H} u\right|_{H} \mathrm{~d} \nu \mathrm{d} s \\
& =\int_{0}^{t} \int_{\Omega}\left(\left|D_{H} T_{\Omega}(s) g\right|_{H}^{2}\right)^{\frac{1}{2}}\left|D_{H} u\right|_{H} \mathrm{~d} \nu \mathrm{d} s \\
& \leq \sqrt{K_{2}} \int_{0}^{t} s^{-\frac{1}{2}} \int_{\Omega}\left(T_{\Omega}(s)|g|^{2}\right)^{\frac{1}{2}}\left|D_{H} u\right|_{H} \mathrm{~d} \nu \mathrm{d} s
\end{aligned}
$$

From the contractivity of $T_{\Omega}(t)$ in $L^{\infty}(\Omega, v)$, for any $t>0$ we deduce

$$
\begin{aligned}
\int_{\Omega} g\left(T_{\Omega}(t) u-u\right) \mathrm{d} v & \leq \sqrt{K_{2}}\|g\|_{L^{\infty}(\Omega, v)} \int_{0}^{t} s^{-\frac{1}{2}} \int_{\Omega}\left|D_{H} u\right|_{H} \mathrm{~d} v \mathrm{~d} s \\
& =2 \sqrt{K_{2} t}\|g\|_{L^{\infty}(\Omega, v)} \int_{\Omega}\left|D_{H} u\right|_{H} \mathrm{~d} \nu .
\end{aligned}
$$

For $u \in B V(\Omega, v)$, from Proposition 6 we get a sequence $u_{n} \in D^{1,2}(\Omega, v)$ converging to $u$ in $L^{2}(\Omega, v)$ with $\lim _{n \rightarrow \infty} \int_{\Omega}\left|D u_{n}\right|_{H} \mathrm{~d} v=\left|D_{v} u\right|(\Omega)$. Thus, putting $u_{n}$ in place of $u$ in (34) and letting $n \rightarrow \infty$ we get

$$
\int_{\Omega} g\left(T_{\Omega}(t) u-u\right) \mathrm{d} v \leq 2 \sqrt{K_{2} t}\|g\|_{L^{\infty}(\Omega, v)}\left|D_{\nu} u\right|(\Omega), \quad g \in L^{\infty}(\Omega, v) .
$$


Finally, taking the supremum on the $g \in L^{\infty}(\Omega, \nu)$ with $\|g\|_{\infty} \leq 1$ we obtain

$$
\int_{\Omega}\left|T_{\Omega}(t) u-u\right| \mathrm{d} v \leq 2 \sqrt{K_{2} t}\left|D_{v} u\right|(\Omega)
$$

whence (33) follows.

Remark 4. Note that condition (32) is equivalent to

$$
\limsup _{t \rightarrow 0^{+}} \frac{1}{\sqrt{t}} \int_{E^{c} \cap \Omega}\left(T_{\Omega}(t) \chi_{E}\right) \mathrm{d} v<\infty .
$$

Indeed, $\left|T_{\Omega}(t) \chi_{E}-\chi_{E}\right|=\left(\chi_{E}-T_{\Omega}(t) \chi_{E}\right) \chi_{E}+\left(T_{\Omega}(t) \chi_{E}-\chi_{E}\right) \chi_{E^{c}}$. The invariance of $T_{\Omega}(t)$ with respect to $v$ in $\Omega$ yields

$$
\begin{aligned}
\int_{\Omega}\left(\chi_{E}-T_{\Omega}(t) \chi_{E}\right) \chi_{E} \mathrm{~d} v & =\int_{\Omega}\left(T_{\Omega}(t) \chi_{E}-\chi_{E} T_{\Omega}(t) \chi_{E}\right) \mathrm{d} v \\
& =\int_{\Omega} \chi_{E^{c}} T_{\Omega}(t) \chi_{E} \mathrm{~d} v
\end{aligned}
$$

Consequently,

$$
\int_{\Omega}\left|T_{\Omega}(t) \chi_{E}-\chi_{E}\right| \mathrm{d} v=2 \int_{\Omega \cap E^{c}} T_{\Omega}(t) \chi_{E} \mathrm{~d} v .
$$

Now, we prove a quasi-converse of Theorem 5 . We start with a preliminary result for bounded functions.

Proposition 7. Under Hypotheses 1, 2, 3 and 4, let $u \in L^{\infty}(X, v)$ be such that

$$
\liminf _{t \rightarrow 0^{+}} \frac{1}{\sqrt{t}} \int_{\Omega} \int_{X}\left|u\left(e^{-t} x+\sqrt{1-e^{-2 t} y}\right)-u(x)\right| \mathrm{d} \gamma(y) \mathrm{d} v(x)=C<\infty .
$$

Then $u \in B V(\Omega, v)$ and $\left|D_{v} u\right|(\Omega) \leq C\left\|Q_{\infty}^{1 / 2}\right\|_{\mathcal{L}(X)} \sqrt{\pi} / 2$.

Proof. We divide the proof into two steps.

Step 1 . Here we prove that for any $v \in C_{b}^{1}(X)$, it holds that

$$
\begin{aligned}
& \lim _{t \rightarrow 0^{+}} \frac{1}{\sqrt{t}} \int_{\Omega} \int_{X} \mid v\left(e^{-t} x+\sqrt{\left.1-e^{-2 t} y\right)}-v(x) \mid \mathrm{d} \gamma(y) \mathrm{d} v(x)\right. \\
& \quad=\frac{2}{\sqrt{\pi}} \int_{\Omega}|D v(x)| \mathrm{d} v(x) .
\end{aligned}
$$

To this aim, we observe that

$$
\begin{aligned}
& K_{v}(t):=\int_{\Omega} \int_{X}\left|v\left(e^{-t} x+\sqrt{1-e^{-2 t}} y\right)-v(x)\right| \mathrm{d} \gamma(y) \mathrm{d} v(x) \\
= & \int_{\Omega} \int_{X}\left|\int_{0}^{t} \frac{\mathrm{d}}{\mathrm{d} r} v\left(e^{-r} x+\sqrt{1-e^{-2 r}} y\right) \mathrm{d} r\right| \mathrm{d} \gamma(y) \mathrm{d} v(x) \\
= & \int_{\Omega} \int_{X}\left|\int_{0}^{t}\left\langle D v\left(e^{-r} x+\sqrt{1-e^{-2 r}} y\right),-e^{-r} x+\frac{e^{-2 r}}{\sqrt{1-e^{-2 r}}} y\right\rangle \mathrm{d} r\right| \mathrm{d} \gamma(y) \mathrm{d} v(x)
\end{aligned}
$$




$$
\begin{aligned}
& \leq \int_{0}^{t} \frac{e^{-r}}{\sqrt{1-e^{-2 r}}} \cdot \\
& \cdot \int_{\Omega} \int_{X}\left|\left\langle D v\left(e^{-r} x+\sqrt{1-e^{-2 r}} y\right),-\sqrt{1-e^{-2 r}} x+e^{-r} y\right\rangle\right| \mathrm{d} \gamma(y) \mathrm{d} \nu(x) .
\end{aligned}
$$

Now, for $r$ fixed we perform the "Gaussian rotation"

$$
(x, y) \mapsto R_{r}(x, y):=\left(e^{-r} x+\sqrt{1-e^{-2 r} y},-\sqrt{1-e^{-2 r}} x+e^{-r} y\right)=:(u, w)
$$

to get, thanks to the invariance of $\gamma$ under $R_{r}$,

$$
\begin{aligned}
K_{v}(t) \leq & \int_{0}^{t} \frac{e^{-r}}{\sqrt{1-e^{-2 r}}} \int_{X} \int_{X}|\langle D v(u), w\rangle| \cdot \\
& \cdot \chi_{\Omega}\left(e^{-r} u-\sqrt{1-e^{-2 r}} w\right) e^{-U\left(e^{-r} u-\sqrt{1-e^{-2 r}} w\right)} \mathrm{d} \gamma(u) d \gamma(w) \\
= & : \int_{X} \int_{X} f_{v}(t, u, w) \mathrm{d} \gamma(w) d \gamma(u) .
\end{aligned}
$$

We claim that

$$
\lim _{t \rightarrow 0^{+}} \frac{1}{\sqrt{t}} \int_{X} \int_{X} f_{v}(t, u, w) \mathrm{d} \gamma(w) d \gamma(u)=C \int_{\Omega}|D v| \mathrm{d} v .
$$

Indeed, by the convexity of $U$ there exist $z \in X$ and $a \in \mathbb{R}$ such that $U(x) \geq\langle x, z\rangle+a$, hence

$$
\begin{aligned}
& \frac{1}{\sqrt{t}} f_{v}(t, u, w) \leq \frac{1}{\sqrt{t}}|D v(u)||w| e^{|\langle z, u\rangle|+|\langle z, w\rangle|+|a|} \int_{0}^{t} \frac{1}{\sqrt{2 r}} \mathrm{~d} r \\
& \quad=\sqrt{2}|D v(u)||w| e^{|\langle z, u\rangle|+|\langle z, w\rangle|+|a|} \in L^{1}(X \times X, \gamma \otimes \gamma), \quad t \in(0,1)
\end{aligned}
$$

and, using De L'Hôpital's rule, for almost every $(u, w) \in \Omega \times X$

$$
\lim _{t \rightarrow 0^{+}} \frac{f_{v}(t, u, w)}{\sqrt{t}}=\sqrt{2} \chi_{\Omega}(u)\langle D v(u), w\rangle e^{-U(u)} .
$$

So by the dominated convergence theorem we obtain

$$
\begin{aligned}
& \limsup _{t \rightarrow 0^{+}} \frac{1}{\sqrt{t}} \int_{\Omega} \int_{X} \mid v\left(e^{-t} x+\sqrt{\left.1-e^{-2 t} y\right)}-v(x) \mid \mathrm{d} \gamma(y) \mathrm{d} v(x)\right. \\
& \leq \frac{2}{\sqrt{\pi}} \int_{\Omega}|D v(u)| \mathrm{d} v(u)
\end{aligned}
$$

where we used that $\int_{X}|\langle D v(u), w\rangle| \mathrm{d} \gamma(w)=\sqrt{2 / \pi}|D v(u)|$. Indeed, using the factorisation $\gamma=\gamma_{1} \otimes \gamma^{\perp}$, where $\gamma_{1}$ is the 1-dimensional standard Gaussian measure on $E=\operatorname{span} D v(u)$, we get

$$
\int_{X}|\langle D v(u), w\rangle| \mathrm{d} \gamma(w)=2 \int_{\{w:\langle D v(u), w\rangle>0\}}\langle D v(u), w\rangle \mathrm{d} \gamma(w)
$$




$$
=2|D v(u)| \int_{E^{\perp}} \int_{0}^{\infty} t \mathrm{~d} \gamma_{1}(t) d \gamma\left(w^{\prime}\right)=\sqrt{2 / \pi}|D v(u)| .
$$

To conclude, consider the family of linear functionals $L_{t}: C_{b}(X \times X) \rightarrow \mathbb{R}, t \in(0,1)$

$$
L_{t} \varphi=\frac{1}{\sqrt{t}} \int_{\Omega} \int_{X} \varphi(x, y)\left(v\left(e^{-t} x+\sqrt{1-e^{-2 t}} y\right)-v(x)\right) \mathrm{d} \gamma(y) \mathrm{d} v(x) .
$$

By (37) we get $\lim \sup _{t \rightarrow 0^{+}}\left\|L_{t}\right\| \leq 2(\sqrt{\pi})^{-1}\|D v\|_{L^{1}(\Omega, v)}$ and arguing as above

$$
\lim _{t \rightarrow 0^{+}} L_{t} \varphi=\sqrt{2} \int_{\Omega} \int_{X} \varphi(x, y)\langle D u(x), y\rangle \mathrm{d} \gamma(y) \mathrm{d} \nu(x)=: L_{0} \varphi .
$$

So $L_{t}$ weakly* converges to $L_{0}$ as $t \rightarrow 0^{+}$and, by lower semicontinuity of the norm we get (36):

$$
\begin{aligned}
\left\|L_{0}\right\| & =\frac{2}{\sqrt{\pi}} \int_{\Omega}|D v(x)| \mathrm{d} v(x) \leq \liminf _{t \rightarrow 0^{+}}\left\|L_{t}\right\| \\
& \leq \limsup _{t \rightarrow 0^{+}}\left\|L_{t}\right\| \leq \frac{2}{\sqrt{\pi}} \int_{\Omega}|D v(x)| \mathrm{d} v(x) .
\end{aligned}
$$

Step 2. For $u \in L^{\infty}(X, v)$, let $\left(u_{j}\right)_{j \in \mathbb{N}} \subseteq C_{b}^{1}(X)$ be such that $u_{j} \rightarrow u$ in $L^{2}(X, v)$, almost everywhere in $X$ and satisfying (35) (thanks to the dominated convergence theorem). Using (36), (6) and (8) we have

$$
\begin{aligned}
& \lim _{t \rightarrow 0^{+}} \frac{K_{u_{j}}(t)}{\sqrt{t}}=\frac{2}{\sqrt{\pi}} \int_{\Omega}\left|D u_{j}\right| \mathrm{d} v=\frac{2}{\sqrt{\pi}\left\|Q_{\infty}^{1 / 2}\right\|_{\mathcal{L}(X)}} \int_{\Omega}\left\|Q_{\infty}^{1 / 2}\right\|_{\mathcal{L}(X)}\left|D u_{j}\right| \mathrm{d} v \\
& \geq \frac{2}{\sqrt{\pi}\left\|Q_{\infty}^{1 / 2}\right\|_{\mathcal{L}(X)}} \int_{\Omega}\left|Q_{\infty}^{1 / 2} D u_{j}\right| \mathrm{d} v=\frac{2}{\sqrt{\pi}\left\|Q_{\infty}^{1 / 2}\right\|_{\mathcal{L}(X)}} \int_{\Omega}\left|Q_{\infty} D u_{j}\right|_{H} \mathrm{~d} v \\
& =\frac{2}{\sqrt{\pi}\left\|Q_{\infty}^{1 / 2}\right\|_{\mathcal{L}(X)}} \int_{\Omega}\left|D_{H} u_{j}\right|_{H} \mathrm{~d} v=\frac{2}{\sqrt{\pi}\left\|Q_{\infty}^{1 / 2}\right\|_{\mathcal{L}(X)}} \int_{\Omega}\left(T_{\Omega}(\sigma)\left|D_{H} u_{j}\right|_{H}\right) \mathrm{d} v \\
& \geq \frac{2}{\sqrt{\pi}\left\|Q_{\infty}^{1 / 2}\right\|_{\mathcal{L}(X)}} e^{\sigma \lambda_{1}^{-1}} \int_{\Omega}\left|D_{H} T_{\Omega}(\sigma) u_{j}\right|_{H} \mathrm{~d} \nu
\end{aligned}
$$

for any $\sigma \in(0,1)$. Now, since the left-hand side of (38) is uniformly bounded from above by the constant $C$, the $L^{1}$-norm of $D_{H} T_{\Omega}(\sigma) u_{j}$ is bounded as well by the same constant for every $j \in \mathbb{N}$ and $\sigma \in(0,1)$, i.e.

$$
e^{\frac{\sigma}{\lambda_{1}}} \int_{\Omega}\left|D T_{\Omega}(\sigma) u_{j}\right| \mathrm{d} \nu \leq C \frac{\sqrt{\pi}}{2}\left\|Q_{\infty}^{1 / 2}\right\|_{\mathcal{L}(X)}, \quad j \in \mathbb{N}, \sigma>0 .
$$

Thus, recalling that $D_{H} T_{\Omega}(\sigma) u_{j}$ converges to $D_{H} T_{\Omega}(\sigma) u$ in $L^{1}(\Omega, v)$ as $j \rightarrow \infty$ (see (7)), letting first $j \rightarrow \infty$ and then $\sigma \rightarrow 0^{+}$and using formula (24) we get that $\left|D_{\nu} u\right|(\Omega) \leq C\left\|Q_{\infty}^{1 / 2}\right\|_{\mathcal{L}(X)} \sqrt{\pi} / 2$.

The following result is a quasi-converse of Theorem 5. In fact, we give a sufficient condition to have $P_{\nu}(E, \Omega)<\infty$ in terms of the short-time behaviour of $T(t)$ and not of $T_{\Omega}(t)$, where $T(t)$ is the semigroup generated by the operator $L$ defined in (31) in $L^{2}(X, v)$. 
Theorem 6. Under Hypotheses 1, 2, 3 and 4, if $E \in \mathcal{B}(X)$ and

$$
C:=\liminf _{t \rightarrow 0^{+}} \frac{1}{\sqrt{t}}\left\|T(t) \chi_{E}-\chi_{E}\right\|_{L^{1}(\Omega, v)}<\infty
$$

then $P_{\nu}(E, \Omega) \leq C\left\|Q_{\infty}^{1 / 2}\right\|_{\mathcal{L}(X)} \sqrt{\pi} / 2$.

Proof. Choosing $u=\chi_{E}$ in (35) and observing that

$$
\int_{\Omega}\left|\int_{X} f(x, y) \mathrm{d} \gamma(y)\right| \mathrm{d} \nu(x)=\int_{\Omega} \int_{X}|f(x, y)| \mathrm{d} \gamma(y) \mathrm{d} \nu(x)
$$

for any $f$ with constant sign, from Proposition 7 we deduce that if

$$
L:=\liminf _{t \rightarrow 0^{+}} \frac{1}{\sqrt{t}}\left\|S(t) \chi_{E}-\chi_{E}\right\|_{L^{1}(\Omega, v)}<\infty
$$

then $P_{v}(E, \Omega) \leq L\left\|Q_{\infty}^{1 / 2}\right\|_{\mathcal{L}(X)} \sqrt{\pi} / 2$. Here $S(t)$ is the Ornstein-Uhlenbeck semigroup in (1). To conclude we prove that condition (40) is equivalent to (39). From the variation-of-constants formula we deduce

$$
(T(t) g)(x)=(S(t) g)(x)-\int_{0}^{t}\left(S(t-\sigma)\left\langle D_{H} U, D_{H} T(\sigma) g\right\rangle_{H}\right)(x) d \sigma,
$$

for every $g \in \mathcal{F} C_{b}(X)$, v-a.e. $x \in X$ and any $t \geq 0$. To prove (41) it suffices that the map $\sigma \mapsto S(t-\sigma)\left\langle D_{H} U, D_{H} T(\sigma) g\right\rangle_{H}$ belongs to $L^{1}((0, t))$ for any $t>0$. To this aim, let us observe that

$$
\int_{X} \int_{0}^{t} S(t-\sigma)\left\langle D_{H} U, D_{H} T(\sigma) g\right\rangle_{H} d \sigma \mathrm{d} v<\infty
$$

for any $g \in \mathcal{F} C_{b}(X)$. Indeed, the Hölder inequality and the contractivity of $S(t)$ in $L^{2}(X, \gamma)$ allow us to write

$$
\begin{aligned}
\int_{X} & \int_{0}^{t} S(t-\sigma)\left\langle D_{H} U, D_{H} T(\sigma) g\right\rangle_{H} d \sigma \mathrm{d} \nu \\
& =\int_{0}^{t} \int_{X} S(t-\sigma)\left\langle D_{H} U, D_{H} T(\sigma) g\right\rangle_{H} \mathrm{~d} \nu d \sigma \\
& \leq \int_{0}^{t}\left\|S(t-\sigma)\left\langle D_{H} U, D_{H} T(\sigma) g\right\rangle_{H}\right\|_{L^{1}(X, v)} d \sigma \\
& \leq\left\|e^{-U}\right\|_{L^{2}(X, \gamma)} \int_{0}^{t}\left\|S(t-\sigma)\left\langle D_{H} U, D_{H} T(\sigma) g\right\rangle_{H}\right\|_{L^{2}(X, \gamma)} d \sigma \\
& \leq\left\|e^{-U}\right\|_{L^{2}(X, \gamma)} \int_{0}^{t}\left\|\left\langle D_{H} U, D_{H} T(\sigma) g\right\rangle_{H}\right\|_{L^{2}(X, \gamma)} d \sigma \\
& \leq \sqrt{K_{2}}\left\|e^{-U}\right\|_{L^{2}(X, \gamma)}\|g\|_{\infty}\left\|D_{H} U\right\|_{L^{2}(X, \gamma ; H)} \int_{0}^{t} \sigma^{-1 / 2} \mathrm{~d} s \\
& =2 \sqrt{K_{2} t}\left\|e^{-U}\right\|_{L^{2}(X, \gamma)}\|g\|_{\infty}\left\|D_{H} U\right\|_{L^{2}(X, \gamma ; H)},
\end{aligned}
$$


where in the last line we used estimate (7) which holds true even in the case $\Omega=X$ and $T_{\Omega}(t)$ replaced by $T(t)$. Hence, formula (41) follows.

Now, integrating (41) in $\Omega$ with respect to $v$ yields

$$
\begin{aligned}
\left\|S(t) \chi_{E}-\chi_{E}\right\|_{L^{1}(\Omega, v)}-H(t) & \leq\left\|T(t) \chi_{E}-\chi_{E}\right\|_{L^{1}(\Omega, v)} \\
& \leq\left\|S(t) \chi_{E}-\chi_{E}\right\|_{L^{1}(\Omega, v)}+H(t)
\end{aligned}
$$

for any $t>0$ with

$$
H(t):=\left|\int_{X} \int_{0}^{t} S(t-s)\left\langle D_{H} U, D_{H} T(s) \chi_{E}\right\rangle_{H} \mathrm{~d} s \mathrm{~d} v\right|, \quad t>0 .
$$

Using estimate (42) with $g=\chi_{E}$ we infer that $\limsup _{t \rightarrow 0^{+}} \frac{H(t)}{\sqrt{t}}<\infty$. This last estimate, together with (43), prove that (40) is equivalent to (39) and the proof is complete.

Funding Open access funding provided by Università del Salento within the CRUICARE Agreement.

Open Access. This article is licensed under a Creative Commons Attribution 4.0 International License, which permits use, sharing, adaptation, distribution and reproduction in any medium or format, as long as you give appropriate credit to the original author(s) and the source, provide a link to the Creative Commons licence, and indicate if changes were made. The images or other third party material in this article are included in the article's Creative Commons licence, unless indicated otherwise in a credit line to the material. If material is not included in the article's Creative Commons licence and your intended use is not permitted by statutory regulation or exceeds the permitted use, you will need to obtain permission directly from the copyright holder. To view a copy of this licence, visit http://creativecommons.org/licenses/ by/4.0/.

Publisher's Note Springer Nature remains neutral with regard to jurisdictional claims in published maps and institutional affiliations.

\section{REFERENCES}

[1] D. Addona, G. Cappa, S. Ferrari, On the domain of elliptic operators defined in subsets of Wiener spaces, Infin. Dimens. Anal. Quantum Probab. Relat. Top., 23 (2020).

[2] D. Addona, G. Menegatti, M. Miranda Jr, BV functions on open domains: the Wiener case and the Fomin differentiable case, Commun. Pure Appl. Anal., 19 (2020), 2679-2711.

[3] L. Ambrosio, N. Fusco, D. Pallara, Functions of bounded variation and free discontinuity problems, OUP Oxford, (2000).

[4] L. Ambrosio, S. Maniglia, M. Miranda Jr, D. Pallara, $B V$ functions in abstract Wiener spaces, J. Funct. Anal., 3 (2015), 212-230.

[5] L. Ambrosio, M. Miranda Jr, D. Pallara, Some fine properties of $B V$ functions on Wiener spaces, Anal. Geom. Metr. Spaces, 3 (2015), 212-230.

[6] L. Angiuli, S. Ferrari, D. Pallara, Gradient estimates for perturbed Ornstein-Uhlenbeck semigroups on infinite-dimensional convex domains, J. Evol. Equ., 19 (2019), 677-715.

[7] V. I. Bogachev, Differentiable measures and the Malliavin calculus, Mathematical Surveys and Monographs, vol. 164, American Mathematical Society, Providence, RI, 2010.

[8] V. I. Bogachev, Gaussian measures, Mathematical Surveys and Monographs, vol. 62, American Mathematical Society, Providence, RI, 1998. 
[9] G. Cappa, S. Ferrari, Maximal Sobolev regularity for solutions of elliptic equations in infinite dimensional Banach spaces endowed with a weighted Gaussian measure, J. Differential Equations, 261 (2016), 7099-7131.

[10] G. Cappa, S. Ferrari, Maximal Sobolev regularity for solutions of elliptic equations in Banach spaces endowed with a weighted Gaussian measure: the convex subset case, J. Math. Anal. Appl., 458 (2018), 300-331.

[11] E. De Giorgi, Su una teoria generale della misura $(r-1)$-dimensionale in uno spazio ad $r$ dimensioni, Ann. Mat. Pura Appl. 36 (1954), 191-213, and also Ennio De Giorgi: Selected Papers, (L. Ambrosio, G. Dal Maso, M. Forti, M. Miranda, S. Spagnolo eds.), Springer, 2006, 79-99. English translation, Ibid., 58-78.

[12] G. Da Prato, A. Lunardi, BV functions in Hilbert spaces. Math. Ann. to appear.

[13] G. Da Prato, J. Zabczyk, Second order partial differential equations in Hilbert spaces, London Mathematical Society Lecture Note Series, vol. 293, Cambridge University Press, Cambridge, 2002.

[14] K.-J. Engel, R. Nagel, A short course on operator semigroups, Universitext, New York, 2006.

[15] M. Fabian, P. Habala, P. Hájek, V. Montesinos, V. Zizler, Banach space theory, CMS Books in Mathematics/Ouvrages de Mathématiques de la SMC, Springer, New York, 2011.

[16] S. Ferrari, Sobolev spaces with respect to a weighted Gaussian measure in infinite dimensions, Infin. Dimens. Anal. Quantum Probab. Relat. Top., 22 (2019).

[17] M. Fukushima, BV functions and distorted Ornstein Uhlenbeck processes over the abstract Wiener space, J. Funct. Anal. 174 (2000), 227-249.

[18] M. Fukushima, M. Hino, On the space of BV functions and a related stochastic calculus in infinite dimensions, J. Funct. Anal., 183 (2001), 245-268.

[19] M. Hino, H. Hucida, Reflecting Ornstein-Uhlenbeck processes on pinned path spaces, Proceedings of RIMS Workshop on Stochastic Analysis and Applications, (2008), 111-128.

[20] M. Ledoux, Semigroup proofs of the isoperimetric inequality in Euclidean and Gauss space, Bull. Sci. Math., 118 (1994), 485-510.

[21] A. Lunardi, M. Miranda Jr, D. Pallara, $B V$ functions on convex domains in Wiener spaces, Potential Anal., 43 (2015), 23-48.

[22] R. O’Donnell, Analysis of Boolean functions, Cambridge University Press, 2014.

[23] M. Röckner, R.C. Zhu, X.C. Zhu, The stochastic reflection problem on an infnite dimensional convex set and BV functions in a Gelfand triple, Ann. Probab., 40 (2012), 1759-1794.

[24] D. Trevisan, BV-regularity for the Malliavin derivative of the maximum of the Wiener process, Electron. Commun. Probab., 18 (2013).

[25] N. N. Vakhania, V. I. Tarieladze, S. A. Chobanyan, Probability distributions on Banach spaces, Mathematics and its applications, vol. 14, Springer Netherlands, D. Reidel Publishing Company, Dordrecht, Holland, 1987.

[26] L. Zambotti, Integration by parts formulae on convex sets of paths and applications to SPDEs with reflection, Probab. Theory Related Fields, 123 (2002), 579-600. 
Luciana Angiuli, Simone Ferrari and Diego Pallara Dipartimento di Matematica e Fisica "Ennio De Giorgi" Università del Salento

Via per Arnesano

73100 Lecce

Italy

E-mail: diego.pallara@unisalento.it

Luciana Angiuli

E-mail: luciana.angiuli@unisalento.it

Simone Ferrari

E-mail: simone.ferrari@unisalento.it

Diego Pallara

INFN, Sezione di Lecce

Via per Arnesano

73100 Lecce

Italy

Accepted: 17 February 2021 